\title{
On the Capacities of Bipartite Hamiltonians and Unitary Gates
}

\author{
Charles H. Bennett, Aram W. Harrow, Debbie W. Leung, and John A. Smolin
}

\begin{abstract}
We consider interactions as bidirectional channels. We investigate the capacities for interaction Hamiltonians and nonlocal unitary gates to generate entanglement and transmit classical information. We give analytic expressions for the entanglement generating capacity and entanglement-assisted one-way classical communication capacity of interactions, and show that these quantities are additive, so that the asymptotic capacities equal the corresponding 1 -shot capacities. We give general bounds on other capacities, discuss some examples, and conclude with some open questions.
\end{abstract}

Index Terms-Communication capacities, entanglement capacities, two-way quantum channels.

\section{INTRODUCTION}

\section{A. Motivation I-Converting Given Interactions Into Abstract} Resources

T HE fundamental physical resource for performing various quantum information processing tasks is the interaction between various quantum systems. These quantum systems can be, for example, individual registers in a quantum computer, or systems possessed by isolated parties. The interactions are Hamiltonians or their discrete-time incarnation as unitary gates that are nonlocal with respect to individual systems. The information processing tasks of interest include converting a given interaction into a universal quantum gate, generating entanglement between remote parties, and communicating classical or quantum information. These tasks output what are considered abstract resources in quantum information theory, such as entanglement and classical communication. The study of the conversions among these various resources and the efficiencies thereof has proved to be a fruitful field.

While our knowledge about the optimal use of a nonlocal interaction to provide the derived resources is far from complete,

Manuscript received September 16, 2002; revised April 4, 2003. This work was supported in part by the NSA under the U.S. Army Research Office (ARO) under Grants DAAG55-98-C-0041 and DAAD19-01-1-06. Correspondence should be sent to A. W. Harrow and D. W. Leung.

C. H. Bennett and J. A. Smolin are with the IBM T. J. Watson Research Center, Yorktown Heights, NY 10598 USA (e-mail: bennetc@watson.ibm.com; smolin@watson.ibm.com).

A. W. Harrow was with the IBM T. J. Watson Research Center, Yorktown Heights, NY 10598 USA. He is now with the Physics Department, Massachusetts Institute of Technology, Cambridge, MA 02139 USA (e-mail: aram@mit.edu).

D. W. Leung was with the IBM T. J. Watson Research Center, Yorktown Heights, NY 10598 USA. She is now with the Institute of Quantum Information, California Institute of Technology, MSC 107-81, Pasadena, CA 91125 USA (e-mail: wcleung@cs.caltech.edu).

Communicated by P. W. Shor, Associate Editor for Quantum Information Theory.

Digital Object Identifier 10.1109/TIT.2003.814935 important progress has been made. References [1]-[4] provide motivating examples on the interconversion of these resources. Interconversion tasks can be classified as follows.

1) Simulation of one nonlocal Hamiltonian or gate by another: methods for doing so are much studied ${ }^{1}$ some of which are optimal under certain circumstances.

2) Generation of entanglement using nonlocal Hamiltonians and gates: Partial results are obtained in [5]-[8].

3) Classical (or quantum) communications using nonlocal Hamiltonians and gates.

4) Performing a nonlocal quantum operation using entanglement and classical communication: This is the converse of the last two tasks. General formalisms for the 1-shot bipartite case and methods for more specific gates are given in [6]. These tasks are related. First, entanglement, forward classical communication, and backward classical communication are strictly incomparable resources: no one of them can be generated even from an infinite supply of the other two. Thus, the capacity of a given interaction to create each of the three resources cannot exceed the amount used to perform the interaction. For example, the CNOT can be simulated using 1 ebit, ${ }^{2}$ one forward, and one backward classical bit [9], so that the entanglement capacity and both forward and backward classical capacities are upper-bounded by 1. Second, the efficiency for one interaction to simulate another provides bounds on the relative efficiency for the interactions to generate resources. For example, any capacity of SWAP is at most three times that of CNOT since the SWAP can be written as three CNOTs.

In this paper, we focus on tasks 2) and 3), and investigate the capacities of a unitary interaction to generate entanglement and

\footnotetext{
${ }^{1}$ The following is a partial list of results on interconversions of interactions: N. Linden, H. Barjat, R. Carbajo, and R. Freeman, Chem. Phys. Lett., vol. 305, pp. 28-34, 1999; arXive e-print quant-ph/9811043. D. W. Leung, I. L. Chuang, F. Yamaguchi, and Y. Yamamoto. Phys. Rev. A, vol. 61, p. 042310, 2000; arXive e-print quant-ph/9904100. J. Jones and E. Knill. J. Magn. Res., vol. 141, pp. 322-325, 1999; arXive e-print quant-ph/9905008. J. L. Dodd, M. A. Nielsen, M. J. Bremner, and R. T. Thew; arXive e-print quant-ph/0106064. P. Wocjan, D. Janzing, and Th. Beth; arXive e-print quant-ph/0106077. D. Leung, J. Mod Opt., vol. 49, pp. 1199-1217, 2002; arXive e-print quant-ph/0107041v2. M. Stollsteimer and G. Mahler, Phys. Rev. A, vo. 64, p. 052301, 2001; arXive e-print quant-ph/0107059v1. N. Khaneja, R. Brockett, and S. J. Glaser, Phys Rev. A, vol. 63, p. 032308, 2001. C. H. Bennett, J. I. Cirac, M. S. Leifer, D. W Leung, N. Linden, S. Popescu, and G. Vidal, Phys. Rev. A, vol. 66, p. 012305 , 2002; arXive e-print quant-ph/0107035. G. Vidal and J. Cirac; arXive e-prin quant-ph/0108076, 0108077. P. Wocjan, M. Roetteler, D. Janzing, and Th. Beth arXive e-print quant-ph/0109063, 0109088. M. A. Nielsen, M. J. Bremner, J. L. Dodd, A. M. Childs, and C. M. Dawson, Phys. Rev. A, vol. 66, p. 022317, 2002 arXive e-print quant-ph/0109064. H. Chen; arXive e-print quant-ph/0109115 G. Vidal, K. Hammerer, and J. I. Cirac; arXive e-print quant-ph/0112168. L. Masanes, G. Vidal, and J. I. Latorre; arXive e-print quant-ph/0202042. W. Dur, G. Vidal, and J. I. Cirac; arXive e-print quant-ph/0112124.

${ }^{2}$ The unit ebit is defined to be the amount of entanglement in the Einstein-Podolsky-Rosen (EPR) state $\frac{1}{\sqrt{2}}(|00\rangle+|11\rangle)$.
} 
perform classical communication. The unitary interaction can be a nonlocal Hamiltonian or gate. We are primarily concerned with the asymptotic limit, when many uses of the gate (or a long duration of the Hamiltonian) are given. We consider an interaction on two $d$-dimensional systems, allow unlimited local operations, local ancillas of arbitrarily large dimensions, and arbitrary input states. We give expressions for the entanglement generating capacity and the entanglement-assisted one-way classical capacity [10]-[13] of an interaction. We show that these quantities are additive in the sense that the amount of entanglement or classical communication generated by $n$ uses of a gate is $n$ times the amount generated by one use.

\section{B. Motivation II-Interactions as Bidirectional Channels}

The capacities of generating entanglement and communication are well studied in the context of a noiseless or noisy channel connecting a sender (Alice) to a receiver (Bob). In this usual model of a quantum channel, a quantum system is physically transported from Alice to Bob, with possible changes (noise) caused by a quantum operation [14] (i.e., a trace-preserving, completely positive, or TCP, linear map). This model of a channel is unidirectional-Bob cannot send information to Alice. However, such unidirectional interactions are a special case of quantum interactions, and in general, a quantum system cannot affect another without being changed itself. For example, the CNOT (defined in the computational basis) operates in reverse direction in the conjugate basis, and transmits an equivalent amount of information in either direction when used in conjunction with other local gates.

In view of this, we generalize the usual model of a quantum channel to take into account the bidirectional nature of a quantum interaction. We define a "bidirectional channel" as a bipartite quantum operation. Alice and Bob each inputs a state to the "bidirectional channel" and receives an output. This work can be viewed as studying the entanglement and classical capacities in bidirectional channels, restricted to the unitary case.

Throughout this paper, a protocol means a procedure that uses a nonlocal gate one or more times, or a nonlocal Hamiltonian for some total amount of time, possibly also consuming and/or producing various amounts of other standard resources, such as entanglement and classical communication in each direction. We always allow unlimited local operations, and we are interested in a protocol's net yield (production minus consumption) of standard resources per use of the gate or per unit interaction time. The protocol can be written as a quantum circuit, and the net effect can be described as a bipartite quantum operation, with bipartite input and output. We call this quantum operation the protocol as well. In general, there is a tradeoff among the yields of various resources when the protocol is varied. For example, CNOT can transmit a classical bit in the forward or backward direction, but not both. As back communication is generic in a bidirectional channel, a protocol using it is generically interactive.

In the next two subsections, we provide more detailed introductions to the two tasks studied in this paper and discuss closely related work.

\section{Entanglement Generating Capacity of Bidirectional Channels}

In [15], the quantum communication capacity of a channel is shown to be equal to its capacity for generating pure entanglement; a greater quantum capacity typically results if two-way classical communication is allowed. Likewise, a bidirectional channel (bipartite quantum operation) can be used to generate entanglement. Simple examples are considered in [1]-[4]. Reference [5] considers the average amount of entanglement created by one use of a nonlocal operation on a distribution of product states. Reference [6] classifies the type of entanglement (bound or distillable) that can be created from product states. Reference [7] considers the optimal 1-shot rate of creating entanglement using an arbitrary 2-qubit Hamiltonian on possibly entangled pure input states without local ancillas. Reference [8] considers the optimal amount of entanglement created by one use of an arbitrary 2-qubit gate on pure product input states without ancillas. References [7], [8] also exhibit examples in which local ancillas increase the amount of entanglement created.

In this paper, we follow the philosophy of [15] and investigate the asymptotic entanglement generating capacity of a bidirectional channel acting on two $d$-dimensional systems. Contrary to previous work [5]-[8] we do not restrict ourselves to qubit systems, we allow arbitrary local ancillas and input states (including entangled or mixed states), and we consider the most general asymptotic protocols. We also consider the effect of many auxiliary resources including classical communication. We restrict our attention to unitary bidirectional channels. We derive the expression for the capacity, show that it is additive, and discuss the optimal protocol.

Leifer, Henderson, and Linden [16] have independently shown, by similar arguments, that the asymptotic entanglement generating capacity on pure input states is an optimization over a 1-shot expression. They also investigate the capacities for many 2-qubit gates with low dimension ancillas both analytically and numerically.

\section{Classical Communication Capacities of Bidirectional Channels}

The classical capacity of an ordinary (unidirectional) quantum channel is, in general, affected by the availability of auxiliary resources, such as entanglement [17] and back communication. For a general noisy quantum channel, the capacity without auxiliary resources is found in [10], [11], and that with unlimited supply of pure entanglement is found in [12], [13]. The capacity for a noiseless quantum channel with unlimited supply of a certain noisy entangled state is found in [18]-[21].

In treating bidirectional channels, we again follow the philosophy of [10]-[13] and consider various asymptotic classical capacities of unitary bidirectional channels of arbitrary dimensions. We allow unlimited local resources, including free instantaneous local operations, and the freedom for Alice and Bob to attach and remove local ancillas. Shared randomness is also given as a resource. Our philosophy is also similar to Shannon's 
study of the classical capacities of classical two-way communication channels [22].

A new ingredient in the case of bidirectional channels is the simultaneous forward and backward communication, resulting in a pair of achievable rates. One can define many classical capacities other than the forward and the backward capacities. Generally, there is a tradeoff between the forward and backward rates.

Our long-term goals are to obtain expressions for these capacities, understand the tradeoff between forward and backward communication, and relate the quantities to other capacities such as the entanglement generating capacity. In this paper, we define various asymptotic capacities of bidirectional channels. We obtain an expression for the one-way (forward or backward) entanglement-assisted classical capacity for any arbitrary nonlocal gate or Hamiltonian, and the protocol achieving it. The asymptotic capacity is achieved by a 1-shot expression, as an optimization over input ensembles for one use of the gate.

We remark that other independent investigations on optimal methods to perform classical communication in low dimensions without entanglement assistance are being conducted [23]-[25].

\section{E. Structure and Assumptions of the Paper}

In the next section, we discuss in detail the problem of entanglement generation, and derive the expression for the entanglement generating capacity for any nonlocal gate. In Section III, we define various classical communication capacities, followed by a derivation of the entanglement-assisted one-way classical capacity for any nonlocal gate in Section IV. We discuss the similarities and differences between the two derivations in Section V. In Section VI, we prove various general bounds relating the capacities for entanglement generation and classical communication. We conclude in Section VIII with open questions and examples of unitary bidirectional channels.

Throughout the paper, we assume the following. Unless otherwise noted, logarithms are in base $2 . O(n)$ and $o(n)$, respectively, denote functions linear and sublinear in $n . U$ denotes a nonlocal gate acting on two $d$-dimensional systems (with shorthand $d \times d$ ) in the possession of Alice and Bob. They have access to the following local resources.

\section{Local ancillas of arbitrarily large but finite dimensions and un- limited local operations.}

We do not consider ancillas of infinite dimensions and do not know if they can be more useful (see Section VIII).

Though we have motivated the discussion with both Hamiltonians and gates, we now argue that it is sufficient to focus on gates only. This is because Hamiltonian capacities are simply gate capacities in the limit of infinitesimal gates, so that any Hamiltonian capacity can be obtained from the corresponding gate capacity. A protocol using a Hamiltonian is similar to one using a gate, with additional freedom on how long each free Hamiltonian evolution can last before being interspersed with local operations. However, different durations of evolution are simply concatenation of different numbers of infinitesimal ones.
Thus, any Hamiltonian capacity $G_{H}$ can be expressed in terms of the corresponding gate capacity $G_{U}$

$$
G_{H}=\lim _{s \rightarrow 0} \frac{1}{s} G_{U=e^{-i H s}} .
$$

\section{ENTANGLEMENT CAPACITY OF BidiRECTIONAL ChanNELS}

\section{A. Main Idea}

Before a formal treatment of the entanglement capacity, we first illustrate our central idea with the following example. Let $E_{e}$ be the entropy of entanglement [31]. Suppose the goal is to increase $E_{e}$ as much as possible. Different uses of $U$ can be used sequentially or in parallel, and be interspersed by local operation and classical communication (LOCC). We allow an arbitrary pure input state with ancillas, possibly entangled over different uses of $U$. What is the optimal strategy? The answer turns out to be very simple. Consider the quantity

$$
\Delta E_{U} \equiv \sup _{|\psi\rangle_{A B A^{\prime} B^{\prime}}} E_{e}\left(U_{A B}|\psi\rangle_{A B A^{\prime} B^{\prime}}\right)-E_{e}\left(|\psi\rangle_{A B A^{\prime} B^{\prime}}\right)
$$

which represents the entanglement generated by optimizing the input state for just one use of $U$. In (1), subscripts label the quantum systems where a state resides or where an operation acts on. Let $\left|\psi_{*}\right\rangle_{A B A^{\prime} B^{\prime}}$ attain the supremum. Then applying individual uses of $U$ to copies of $\left|\psi_{*}\right\rangle_{A B A^{\prime} B^{\prime}}$ is asymptotically optimal. This is because the total increase in $E_{e}$ in any asymptotic protocol is at most the sum of the increases due to each use of $U$, and each is no greater than $\Delta E_{U}$.

In the following, we will develop this idea rigorously in the most general setting. We consider mixed input states and different entanglement measures, and analyze the roles of various auxiliary resources.

\section{B. Definitions and Summary of Results}

The entanglement capacity of a gate $U$ can only be defined when the entanglement measures for the input and output and the available auxiliary resources are specified.

Traditionally, entanglement is a qualitative phenomenon. The theory of quantifying entanglement is not complete, though much progress has been made $^{3}$ ([26], [27] give informative reviews). Based on the transformation properties of entangled states, measures of entanglement are defined which are very different in the asymptotic and nonasymptotic regimes. Different measures in the same regime can also be inequivalent.

The entanglement generated by a protocol $\mathcal{P}$ on an input $\rho$ is intuitively

$$
E_{\text {out }}(\mathcal{P}(\rho))-E_{\text {in }}(\rho)
$$

where $E_{\text {in }}, E_{\text {out }}$ are the input and output entanglement measures specified in the problem. We can now define the entanglement capacity of $U$.

\footnotetext{
${ }^{3}$ A partial list includes [28], [29] as well as the following: S. Popescu, and D. Rohrlich, Phys. Rev. A, vol. 56, 1997; P3319. arXive e-print quant-ph/9610044. V. Vedral and M. B. Plenio, Phys. Rev. A., vol. 57, pp. 1619-1633, 1998; arXive e-print quant-ph/9707035. A. Uhlmann, Open Sys. Inf. Dyn., vol. 5, p. 209, 1998; arXive e-print quant-ph/9701014. M. Horodecki, P. Horodecki, R. Horodecki, Phys. Rev. Lett., vol. 84 p. 2014, 2000; arXive e-print quant-ph/9908065.
} 
Definition 1: The $t$-shot entanglement capacity of $U$ is the maximum amount of entanglement generated per use of $U$ by any protocol $\mathcal{P}_{t}$ that uses $U t$ times, auxiliary resources labeled by $r$, and local resources specified in Section I-E (ancillas of arbitrary but finite dimensions and unlimited local operations). We consider two possible $t$-shot capacities, depending on the allowed input state:

1) when the input is restricted to be a product state (without loss of generality $|00\rangle$, since Alice and Bob can locally transform any product state into any other product state)

$E_{\text {in } \rightarrow \text { out }, U}^{(t, \emptyset, r)} \equiv \sup _{\mathcal{P}_{t}} \frac{1}{t}\left[E_{\text {out }}\left(\mathcal{P}_{t}(|00\rangle\langle 00|)\right)-E_{\text {in }}(|00\rangle\langle 00|)\right]$

where the superscript $\emptyset$ denotes "starting from nothing," and

2) when there is no restriction on the input state

$$
E_{\text {in } \rightarrow \text { out }, U}^{(t, *, r)} \equiv \sup _{\mathcal{P}_{t}} \sup _{\rho} \frac{1}{t}\left[E_{\text {out }}\left(\mathcal{P}_{t}(\rho)\right)-E_{\text {in }}(\rho)\right]
$$

where the superscript $*$ denotes an optimization over all possible input states.

The corresponding asymptotic capacities are

$$
\begin{aligned}
& E_{\text {in } \rightarrow \text { out }, U}^{(\emptyset, r)} \equiv \lim _{t \rightarrow \infty} E_{\text {in } \rightarrow \text { out }, U}^{(t, \emptyset), r} \\
& E_{\text {in } \rightarrow \text { out }, U}^{(*, r)} \equiv \lim _{t \rightarrow \infty} E_{\text {in } \rightarrow \text { out }, U}^{(t, *, r)}
\end{aligned}
$$

In our notation, we assume that an entanglement measure is written as $E_{x}$ where the subscript $x$ labels the measure. For example, in $E_{\text {in }}, E_{\text {out }}$, and the notation for the above capacities, the "in" and "out" are placeholders for the entanglement measures being referred to. Whenever $E_{\text {in }}=E_{\text {out }}=E_{x}$ we simplify the notation of the capacity to $E_{x, U}^{(\cdots)}$. Finally, an arbitrary entanglement measure is written as $E$ without the subscript, and the capacity is written as $E_{U}^{(\cdots)}$.

By definition, $E_{\mathrm{in} \rightarrow \text { out }, U}^{(t, \emptyset, r)} \leq E_{\mathrm{in} \rightarrow \text { out }, U}^{(t, *, r)}$. The capacity $E_{\text {in } \rightarrow \text { out, } U}^{(t, *)}$ has an operational meaning that a supply of the initial state $\rho$ is available at a price $E_{\text {in }}(\rho)$. This is a resource, because the ability to create $\rho$ with an average cost $E_{\text {in }}(\rho)$ is generally not guaranteed (unless $E_{\text {in }}$ is the entanglement cost [28]). We refer to this as "the resource $*$ " throughout the paper. In contrast, no such resource is assumed in the capacity $E_{\text {in } \rightarrow \text { out }}^{(t, \emptyset)}$

Since we are interested in asymptotic capacities, we are primarily concerned with asymptotic measures. These include the entanglement cost $E_{c}$ [28] and the distillable entanglement $E_{d}$ [15]. We also study the entanglement of formation $E_{f}$ [15], which is closely related to $E_{c}$. All of these measures coincide with the entropy of entanglement $E_{e}$ on pure states. As our results apply to more general measures, and may be useful in other contexts, we follow an abstract approach [26], [27], which requires more technicalities in our arguments. However, the essence can be made clear by relating to the discussion of the special case in Section II-A, and we leave this step to the readers as an exercise.

The auxiliary resources can be divided into three types according to their quantities. The first type is given in an amount that is negligible or can be recovered at the end of the protocol.
For example, a sublinear (in the number of uses of $U$ ) amount of resources in the asymptotic case are negligible, and catalytic resources in the 1-shot case are used and regenerated (for example, see [29]). We need not consider these resources. In the 1 -shot case, catalytic resources are a subset of the resource $*$ and need not be treated separately. In the asymptotic case, the sublinear amount of any resource can be produced at a vanishing average cost and it does not affect the asymptotic capacity. This is because any nonlocal gate has nonzero capacity to create pure entanglement and to perform classical communication (see Section VI) from which any other resource can be produced. The second type of resources are at least linear in the number of uses of $U$. To consider these resources is an important open question, but it is out of the scope of the present paper. The third type of resources are unlimited and free. In the context of generating entanglement, we focus on the auxiliary resource of unlimited two-way classical communication, labeled by "cc."

Our results can be summarized in terms of the entanglement capacities just defined.

- In Section II-C, we show that if $E_{\text {in }}=E_{\text {out }}=E$ and $E$ is nonincreasing under LOCC, $E_{U}^{(1, *, \mathrm{cc})} \geq E_{U}^{(*, \mathrm{cc})}$. Thus, given the resource $*$, the 1-shot capacity is no less than the asymptotic capacity. We give a sufficient condition for additivity, $E_{U}^{(1, *, c c)}=E_{U}^{(*, c c)}$, and we describe an optimal asymptotic protocol that does not require classical communication (thus, $\left.E_{U}^{(t, *, \mathrm{cc})}=E_{U}^{(t, *)}\right)$. Additivity holds for many measures including $E=E_{c}, E_{f}, E_{d}$.

- In Section II-D, we consider $E=E_{c}$, and we show that $E_{c, U}^{(*, c c)}=E_{c, U}^{(\emptyset, c c)}=E_{c, U}^{(\emptyset)}$ by describing an explicit protocol. We show the same for $E_{f}$. In other words, * or cc does not increase the asymptotic capacity of $E_{c, f}$, which can be attained without classical communication or a supply of the optimal input.

- In Section II-E, we consider the maximum gain of pure entanglement. This is given by $E_{\mathrm{c} \rightarrow \mathrm{d}, U}^{(t, *, \mathrm{cc})}$, and we show that it is equal to $E_{\mathrm{c}, U}^{(t, *, \mathrm{cc})}$. Thus, the optimal protocol in Section II-D applies, without the need of resources $*$ or cc.

\section{Expression for $E_{U}^{(*, c c)}$ When $E_{\mathrm{in}}=E_{\mathrm{out}}=E$}

Throughout this subsection, $E_{\text {in }}=E_{\text {out }}=E$ and both resources $*$, cc are available. Let

$$
\left|\Phi_{n}\right\rangle=\frac{1}{\sqrt{n}} \sum_{i=1}^{n}|i\rangle_{A}|i\rangle_{B}
$$

be the $n \times n$ maximally entangled state shared between Alice and Bob. Unless otherwise stated, $E$ satisfies the following assumptions, but is otherwise arbitrary:

A1. $E=0$ for product states.

A2. $E$ is invariant under local unitaries.

A3. $E$ is nonincreasing under LOCC.

A4. $\forall_{\rho} E\left(\rho \otimes\left|\Phi_{n}\right\rangle\left\langle\Phi_{n}\right|\right)=E(\rho)+E\left(\left|\Phi_{n}\right\rangle\right)$.

A1-A3 are basic axioms for entanglement measures, while A4 is needed to define the "net" amount of entanglement generated by a protocol. Generally, we do not assume $E$ is normalized $\left(\forall_{n} E\left(\left|\Phi_{n}\right\rangle\right)=\log n\right)$ and will state the assumption explicitly when it is needed. 
We first state a lemma based on the following simple observation [3], [4]. Alice and Bob can implement $U$ if Alice teleports her input to Bob, who applies $U$ locally in his own laboratory and teleports her output back to her. This consumes two copies of $\left|\Phi_{d}\right\rangle$ and $2 \log d$ bits of classical communication in each direction.

Lemma 1: $\forall_{t} E_{U}^{(t, *, c c)} \leq 2 E\left(\left|\Phi_{d}\right\rangle\right)$. Thus, $E_{U}^{(*, c c)} \leq$ $2 E\left(\left|\Phi_{d}\right\rangle\right)$. If furthermore $E$ is normalized, $E_{U}^{(*, c c)} \leq 2 \log d$.

Proof: For any protocol with $t$ uses of $U$ and LOCC, modify it by replacing each use of $U$ with its double teleportation implementation. Let $\rho_{\text {in }}$ and $\rho_{\text {out }}$ be the input and output of the original protocol. The modified protocol uses only LOCC and has input $\rho_{\text {in }} \otimes\left|\Phi_{d}\right\rangle\left\langle\left.\Phi_{d}\right|^{\otimes 2 t}\right.$ and output $\rho_{\text {out }}$. Applying A3 and $\mathrm{A} 4$ to the modified protocol

$$
2 t E\left(\left|\Phi_{d}\right\rangle\right) \geq E\left(\rho_{\text {out }}\right)-E\left(\rho_{\text {in }}\right) .
$$

We now proceed to prove Theorem 1, which says that the asymptotic capacity is equal to the 1-shot capacity given the resources $*$ and cc. This is done by proving two separate inequalities, each is referred to as a half of the theorem.

Theorem 1 (First Half): $E_{U}^{(t, *, \mathrm{cc})} \leq E_{U}^{(1, *, \mathrm{cc})}$.

Proof: Since LOCC operations cannot increase entanglement, the best 1-use protocol has the form

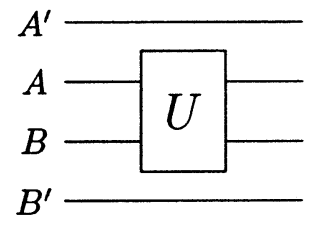

The only optimization is over the initial state, and thus

$$
E_{U}^{(1, *, c c)}=\sup _{\rho_{A A^{\prime} B B^{\prime}}} E\left(U_{A B} \rho_{A A^{\prime} B B^{\prime}} U_{A B}^{\dagger}\right)-E\left(\rho_{A A^{\prime} B B^{\prime}}\right) .
$$

In (7) and throughout the paper, the subscripts of an operator denote the systems being acted on. As an aside, $E_{U}^{(1, *, c c)}=$ $E_{U}^{(1, *)}$ since no classical communication is used in the protocol depicted in (6).

Now, consider any protocol with LOCC and $t$ uses of $U$. Without loss of generality, we can divide the circuit into time steps each having either one use of $U$ or only LOCC operations. The entanglement can only increase in the $t$ time steps with $U$ and each is described by (6), by defining the ancillas $A^{\prime}$ and $B^{\prime}$ to include all registers not acted on by $U$ in that time step. Thus, the total amount of entanglement generated by the protocol is no more than $t E_{U}^{(1, *)}$, and

$$
E_{U}^{(t, *, \mathrm{cc})} \leq E_{U}^{(1, *)}=E_{U}^{(1, *, \mathrm{cc})} .
$$

We now consider sufficient conditions for additivity, $E_{U}^{(t, *, \mathrm{cc})}=E_{U}^{(1, *, \mathrm{cc})}$. We say that $E$ is weakly additive on $\rho$ if $E\left(\rho^{\otimes n}\right)=n E(\rho)$. E is weakly additive if it is so on all $\rho$. We say that $E$ is strongly additive on $\rho_{1}$ if

$$
\forall_{\rho_{2}} E\left(\rho_{1} \otimes \rho_{2}\right)=E\left(\rho_{1}\right)+E\left(\rho_{2}\right) .
$$

$E$ is strongly additive if

$$
\forall_{\rho_{1}, \rho_{2}} E\left(\rho_{1} \otimes \rho_{2}\right)=E\left(\rho_{1}\right)+E\left(\rho_{2}\right) .
$$

Weak and strong subadditivity and superadditivity are defined by replacing the equality in the corresponding additivity definitions by the inequalities $\leq$ and $\geq$, respectively.

Theorem 1 (Second Half): If $E$ is weakly additive or subadditive on the optimal input in (7) and is weakly additive or superadditive on the optimal output, then $E_{U}^{(t, *, \mathrm{cc})} \geq E_{U}^{(1, *, \mathrm{cc})}$.

Proof: Consider the $t$-use protocol that repeats the 1-use protocol in (6) $t$ times, each acting on a separate copy of the optimal input. The entanglement generated is at least $t E_{U}^{(1, *, c c)}$, and $E_{U}^{(t, *, \mathrm{cc})} \geq E_{U}^{(1, *, \mathrm{cc})}$.

Note that any measure which is weakly additive and is nonincreasing under LOCC satisfies both halves of Theorem 1 and $E_{U}^{(*, c c)}=E_{U}^{(1, *, c c)}$. Weak additivity is not an axiomatic property of entanglement, and needs to be checked for individual measures before applying the second half of Theorem 1. On the other hand, Theorem 1 does hold for most commonly used measures. Examples include $E_{c}, E_{d}{ }^{4}[30]$ and the Rényi entropy (which includes the mixed-state generalizations of the logarithm of the Schmidt number and $E_{e}$ as special cases). It is an open problem whether $E_{f}$ is weakly additivity, however, we will prove that the second half of Theorem 1 still holds for $E=E_{f}$.

In general, we say that "Theorem 1 holds" whenever both halves of Theorem 1 hold. Equation (7) then provides an explicit expression for $E_{U}^{(*, c c)}$ achieved by repeating the 1-use protocol. In fact, the protocol only requires a supply of the optimal input (the resource $*$ ) but not classical communication, and $E_{U}^{(*, c c)}=E_{U}^{(*)}$. In the following, we prove some lemmas for $E_{U}^{(1, *, \mathrm{cc})}$. We discuss how to obtain this supply of optimal input in Section II-D.

In the expression for $E_{U}^{(1, *, c c)}$ in (7), the supremum is taken over finite but arbitrarily large dimensional ancillas $A^{\prime} B^{\prime}$. This can also be viewed as a limiting quantity as the ancilla dimensions increase.

Lemma 2: Suppose we restrict to $n$-dimensional $A^{\prime}, B^{\prime}$ in (7), and denote the subsequent maximization by $e_{n}$. Then, $\lim _{n \rightarrow \infty} e_{n}=E_{U}^{(1, *, c c)}$.

Proof: The sequence $\left\{e_{n}\right\}$ is increasing and bounded above by Lemma 1. Thus, $\forall_{\epsilon>0} \exists n_{o}$ such that $e_{n_{o}} \geq E_{U}^{(1, *, \mathrm{cc})}-\epsilon$.

Lemma 2 provides one possible way to estimate the supremum in (7) suitable for numerical approaches.

We say that " $E_{U}^{(1, *, c c)}-\epsilon$ can be attained on an input $\rho$ " if the 1-shot protocol generates an amount of entanglement $E_{U}^{(1, *, c c)}-\epsilon$ on the input $\rho$. The next two lemmas show that the optimal input for the 1-shot capacity in (7) can be chosen pure for the specific measures $E_{f}$ and $E_{c}$.

Lemma 3: For any $\epsilon>0, E_{f, U}^{(1, *, c c)}-\epsilon$ can be attained on a pure input state.

\footnotetext{
${ }^{4} E_{d}$ is weakly additive and nonincreasing under LOCC by definition. There is doubt on its convexity. [30], However, violation of convexity does not imply $E_{d}$ can be increased by LOCC.
} 
Proof: Let $\rho_{A A^{\prime} B B^{\prime}}$ be a state attaining the supremum in (7) to within $\epsilon$. We omit the system label when it is $A A^{\prime} B B^{\prime}$ in Lemmas 3 and 4. Let $\rho=\sum_{i} \lambda_{i}\left|\psi_{i}\right\rangle\left\langle\psi_{i}\right|$ be an optimal decomposition so that $E_{f}(\rho)=\sum_{i} \lambda_{i} E_{f}\left(\left|\psi_{i}\right\rangle\right)$. Then

$$
\begin{aligned}
E_{f, U}^{(1, *, \mathrm{cc})}-\epsilon & \leq E_{f}\left(U_{A B} \rho U_{A B}^{\dagger}\right)-E_{f}(\rho) \\
& \leq \sum_{i} \lambda_{i}\left[E_{f}\left(U_{A B}\left|\psi_{i}\right\rangle\right)-E_{f}\left(\left|\psi_{i}\right\rangle\right)\right] \\
& \leq \max _{i}\left[E_{f}\left(U_{A B}\left|\psi_{i}\right\rangle\right)-E_{f}\left(\left|\psi_{i}\right\rangle\right)\right] .
\end{aligned}
$$

The second inequality is obtained by applying convexity of $E_{f}$ to the first term, and the definition of the optimal decomposition in the second term. Thus, $E_{f, U}^{(1, *, c c)}-\epsilon$ can be attained on a pure input state.

Lemma 4: For any $\epsilon>0, E_{c, U}^{(1, *, c c)}-\epsilon$ can be attained on a pure input state.

Proof: Let $\rho$ attain the supremum in (7) up to $\epsilon / 2$. That is,

$$
E_{c, U}^{(1, *, c c)}-\frac{\epsilon}{2} \leq E_{c}\left(U_{A B} \rho U_{A B}^{\dagger}\right)-E_{c}(\rho) .
$$

For any $\delta>0, \exists m$ such that $\frac{1}{m} E_{f}\left(\rho^{\otimes m}\right)-E_{c}(\rho) \leq \delta$ [28]. Substitute this into (9) with $\delta=\epsilon / 2$

$$
E_{c, U}^{(1, *, c c)}-\frac{\epsilon}{2} \leq E_{c}\left(U_{A B} \rho U_{A B}^{\dagger}\right)-\frac{1}{m} E_{f}\left(\rho^{\otimes m}\right)+\frac{\epsilon}{2} .
$$

Using weak additivity of $E_{c}$ and the fact $E_{f} \geq E_{c}$, the first term in the right-hand side (RHS) of (10) can be rewritten as

$$
E_{c, U}^{(1, *, c c)}-\epsilon \leq \frac{1}{m}\left[E_{f}\left(U_{A B}^{\otimes m} \rho^{\otimes m} U_{A B}^{\dagger \otimes m}\right)-E_{f}\left(\rho^{\otimes m}\right)\right] .
$$

But the expression in the bracket represents the entanglement of formation generated by a certain $m$-use protocol, and is not greater than $m E_{f, U}^{(1, *, c c)}$, by the first half of Theorem 1 . Together with Lemma 3

$$
E_{c, U}^{(1, *, c c)}-\epsilon \leq \sup _{|\psi\rangle} E_{f}\left(U_{A B}|\psi\rangle\right)-E_{f}(|\psi\rangle) .
$$

Finally, we replace $E_{f}$ by $E_{c}$ on the RHS since they coincide on pure states

$$
E_{c, U}^{(1, *, c c)}-\epsilon \leq \sup _{|\psi\rangle} E_{c}\left(U_{A B}|\psi\rangle\right)-E_{c}(|\psi\rangle)
$$

which proves our claim.

When Theorem 1 holds, lemmas about $E_{U}^{(1, *, c c)}$ hold for $E_{U}^{(*, c c)}$ as well. This fact, together with Lemmas 2-4, lead to many useful corollaries. These are given with numbers matching those of the corresponding lemmas.

Corollary 2: If Theorem 1 holds for $E$, then $E_{U}^{(*, c c)}$ is achievable using the 1-use protocol in (6) with sufficiently large-dimensional $A^{\prime}, B^{\prime}$.

Corollary 3.1: Since $E_{f}$ is strongly additive on pure states, Lemma 3 implies that Theorem 1 holds for $E_{f}$.

Corollary 3.2, 4.1: Corollary 3.1 and Lemma 4 imply that $\forall_{\epsilon>0}, E_{f, U}^{(*, c c)}-\epsilon$ and $E_{c, U}^{(*, c c)}-\epsilon$ are each attainable with the 1 -shot protocol in (6) on a pure input state.

Corollary $3+4: E_{f, U}^{(1, *, \mathrm{cc})}=E_{c, U}^{(1, *, \mathrm{cc})}$ with common pure optimal inputs. Same for $E_{f, U}^{(*, c c)}=E_{c, U}^{(*, c c)}$.
It is unclear whether $E_{d, U}^{(1, *, c c)}-\epsilon$ can be attained on a pure state. Convexity in $E_{f, c}$ is required in our proofs of Lemmas 3 and 4, but unlike $E_{f}$ and $E_{c}, E_{d}$ may not be convex [30].

Note that Theorem 1 is concerned with weak additivity of the entanglement capacity of bidirectional channels, i.e., the protocol uses only one type of nonlocal gate. We can consider strong additivity when different types of nonlocal gates are available:

Theorem 1S (First Half): For a protocol with $n_{i}$ uses of the gate $U_{i}$, the maximum amount of entanglement generated (given $*, \mathrm{cc})$ is no more than $\sum_{i} n_{i} E_{U_{i}}^{(1, *)}$.

Theorem $1 S$ (Second Half): If $E$ is strongly additive or subadditive on the optimal input and strongly additive or superadditive on the optimal output for each $U_{i}$, then repeating $n_{i}$ times the 1-shot protocol for $U_{i}$ for each $i$ generates an amount of entanglement no less than $\sum_{i} n_{i} E_{U_{i}}^{(1, *, c c)}$.In particular, Theorem 1S holds for $E=E_{f, c}$, and the entanglement capacities are strongly additive given $*$ and cc.

\section{Auxiliary Resources are Unnecessary When $E=E_{c}$ or $E_{f}$}

In this subsection, we show that the resource $*$ is unnecessary in the optimal asymptotic protocol in the previous subsection (repeating the optimal 1-shot protocol) for the specific measures $E_{\text {in }}=E_{\text {out }}=E_{c, f}$.

By Lemma 4, when $E=E_{c}$, the optimal input and output of the 1-shot protocol are pure. The amount of entanglement $E_{c, U}^{(*, c c)}-\epsilon=E_{c, U}^{(1, *, c c)}-\epsilon$ can be generated by adapting an argument in [7]. The protocol first creates $m$ copies of the pure optimal input $|\psi\rangle_{A A^{\prime} B B^{\prime}}^{\otimes m}$ (inefficiently), and then repeats the cycle: 1) apply $\left.U^{\otimes m}, 2\right)$ "concentrate" [31] the outputs to EPR pairs, and 3) dilute some of the EPR pairs to form $|\psi\rangle_{A A^{\prime} B B^{\prime}}^{\otimes m}$ [32]-[35]. For large $m$, dilution and concentration take $O(\sqrt{m})$ classical communication and waste $O(\sqrt{m})$ amount of entanglement [34], [35]; both can be supplied by an additional $O(\sqrt{m})$ uses of $U$-negligible for sufficiently large $m$. The cost of creating the first $m$ copies of the pure optimal input inefficiently is also negligible when the cycle is repeated sufficiently many times. The same argument holds for $E=E_{f}$.

$$
\text { Corollary 3.3, 4.2: } \quad E_{c, U}^{(*, c c)}=E_{c, U}^{(\emptyset)} \text { and } E_{f, U}^{(*, c c)}=E_{f, U}^{(\emptyset)} \text {. }
$$

The asymptotic entanglement capacity for $E=E_{c, f}$ under the most general setting in Section II-B can be generated without initial entanglement and without $*$ or cc. The core part of the optimal protocol is basically an 1-shot-tensor product of the optimal 1-shot protocol. The only collective steps, entanglement concentration and dilution, are auxiliary.

Since no initial entanglement is required for the optimal asymptotic generation of $E_{f}$ and $E_{c}$, one can relate the asymptotic entanglement capacities to the Schmidt number of $U$. Any bipartite pure state can be written as $\sum_{i} \lambda_{i}\left|\phi_{i}\right\rangle_{A}\left|\eta_{i}\right\rangle_{B}$, where $\lambda_{i}>0, \sum_{i} \lambda_{i}^{2}=1$, and $\left\{\left|\phi_{i}\right\rangle\right\},\left\{\left|\eta_{i}\right\rangle\right\}$ are orthonormal sets of states. Likewise, any $d \times d$ bipartite unitary gate can be written as $\sum_{i} \lambda_{i} V_{i A} \otimes W_{i B}$, where $\lambda_{i}>0, \sum_{i} \lambda_{i}^{2}=d^{2}$, and $\left\{V_{i A}\right\},\left\{W_{i B}\right\}$ are sets of operators orthonormal under the trace norm. The Schmidt number of the bipartite pure state or gate [14], [36], denoted as $\operatorname{Sch}(\cdot)$, is the unique number of 
terms in the above "Schmidt decomposition." The $\lambda_{i}$ are called the Schmidt coefficients. We will repeatedly use the fact that the Schmidt number of a state is nonincreasing under LOCC and that $\operatorname{Sch}(U|\psi\rangle) \leq \operatorname{Sch}(U) \operatorname{Sch}(|\psi\rangle)$ (see [36, Ch. 6.4.2]).

Corollary 3.4, 4.3: $E_{c, U}^{(*, \mathrm{cc})}=E_{f, U}^{(*, \mathrm{cc})} \leq \log \operatorname{Sch}(U)$.

Proof: Since $E_{c, U}^{(*, c c)}$ and $E_{f, U}^{(*, c c)}$ can be achieved without initial entanglement, the initial state has Schmidt number 1 , and the final state of a $t$-use protocol has Schmidt number $\leq \operatorname{Sch}(U)^{t}$. Hence, the output entropy of entanglement is $\leq t \log \operatorname{Sch}(U)$, and

$$
E_{c, U}^{(*, \mathrm{cc})}=E_{f, U}^{(*, \mathrm{cc})} \leq \log \operatorname{Sch}(U) .
$$

Corollary 3.5, 4.4: $E_{c, U}^{(1, \emptyset)}=E_{f, U}^{(1, \emptyset)} \geq-\sum_{i} \lambda_{i}^{2} \log \lambda_{i}^{2}$ where $\left\{\lambda_{i}\right\}$ are the Schmidt coefficients of $U$.

Proof: This is the entanglement generated when

$$
|\psi\rangle_{A A^{\prime} B B^{\prime}}=\left|\Phi_{d}\right\rangle_{A A^{\prime}}\left|\Phi_{d}\right\rangle_{B B^{\prime}}
$$

in (7) [38].

Interested readers can repeat the preceding analysis for other measures. It holds for $E=E_{d}$ if the optimal input $\rho$ is pure or if $\rho$ satisfies $E_{c}(\rho)=E_{d}(\rho)$ (by replacing concentration with distillation of the optimal output and replenishing the optimal input $\rho$ using $E_{d}(\rho)$ EPR pairs per copy of $\rho$ and classical communication (see the Appendix)).

\section{E. Different Input and Output Entanglement Measures}

Each choice of entanglement measures for the input and output can be given an operational meaning. We consider the important example of creating EPR pairs in this subsection, which requires different entanglement measures for the input and output. Alice and Bob fabricate the possibly mixed optimal input state and distill entanglement from the output. Thus, the appropriate choices for the input and output entanglement measures are the entanglement cost $E_{c}$ and the distillable entanglement $E_{d} \cdot{ }^{5}$ Let $\mathcal{P}_{t}$ denote an optimal $t$-shot protocol and the corresponding quantum operation, and let $\rho$ be the optimal input to within $\epsilon$ (again we omit the system label $\left.A A^{\prime} B B^{\prime}\right)$. Then

$$
\begin{aligned}
E_{c \rightarrow d, U}^{(t, *, c c)}-\epsilon & \leq \frac{1}{t}\left[E_{d}\left(\mathcal{P}_{t}(\rho)\right)-E_{c}(\rho)\right] \\
& \leq \frac{1}{t}\left[E_{c}\left(\mathcal{P}_{t}(\rho)\right)-E_{c}(\rho)\right] \\
& \leq \sup _{|\psi\rangle} E_{c}\left(U_{A B}|\psi\rangle\right)-E_{c}(|\psi\rangle) \\
& =\sup _{|\psi\rangle} E_{d}\left(U_{A B}|\psi\rangle\right)-E_{c}(|\psi\rangle) \\
& =E_{c, U}^{(1, *)} \leq E_{c \rightarrow d, U}^{(1, *)}
\end{aligned}
$$

where we have used Corollary 4 in Section II-C to obtain (14). This means that the asymptotic capacity to create EPR pairs is

$$
E_{c \rightarrow d, U}^{(t, *, c c)}=\sup _{|\psi\rangle} E_{c}\left(U_{A B}|\psi\rangle\right)-E_{c}(|\psi\rangle)
$$

and the protocol in Section II-D is optimal for creating EPR pairs even in the most general setting described in Section II-B.

\footnotetext{
${ }^{5}$ Note that $t$ is finite, but we have chosen the asymptotic measures $E_{c}$ and $E_{d}$. We are mainly interested in protocols with large $t$, with the understanding that the 1 -shot capacity $E_{c \rightarrow d, U}^{(1, *)}$ is achieved with collective pre- and postprocessing.
}

Furthermore, since the optimal output is pure, and $E_{d}, E_{c}$ are strongly additive on pure states, strong additivity (Theorem 1S) holds when different types of gates are given.

\section{F. Summary}

We summarize our results obtained so far:

1) $E_{U}^{(1, *)}=E_{U}^{(1, *, c c)} \geq E_{U}^{(*, c c)}$ for all $E$;

2) $E_{U}^{(1, *)}=E_{U}^{(*, c c)}=E_{U}^{(*)}$ for all $E$ weakly additive on the 1-shot optimal input and output;

3) $E_{U}^{(1, *)}=E_{U}^{(\emptyset)}$ for $E=E_{c, f}$;

4) $E_{c, U}^{(1, *)}=E_{c \rightarrow d, U}^{(*, c c)}$.

In particular, when $E_{\text {in }}=E_{\text {out }}=E_{f}$, or when $E_{\text {in }}=E_{c}$, and $E_{\text {out }}=E_{c}$ or $E_{d}$, the asymptotic capacities become independent of the availability of $*$ and cc, and they are all equal to $E_{e, U}^{(1, *)}=\Delta E_{U}$ in (1). The only capacity mentioned above that is different from $\Delta E_{U}$ is $E_{\mathrm{in} \rightarrow \text { out }, U}^{(1, \emptyset)}$. We will study these two capacities in Sections VI and VIII in more detail.

As an aside, when $E=E_{c}$ (or $E_{f}$ ), if $E_{c, U}^{(1, \emptyset)}<E_{c, U}^{(\emptyset)}$, then $E_{c, U}^{(t, \emptyset)}<E_{c, U}^{(\emptyset)}$ for all finite $t$. This is because

$$
\begin{aligned}
t E_{c, U}^{(t, \emptyset)} & \leq E_{c, U}^{(1, \emptyset)}+(t-1) E_{c, U}^{(t-1, *)} \\
& =E_{c, U}^{(1, \emptyset)}+(t-1) E_{c, U}^{(\emptyset)}<t E_{c, U}^{(\emptyset)} .
\end{aligned}
$$

\section{Classical Capacities of Bidirectional Channels}

If Alice and Bob have access to a nonlocal gate $U$ to couple their systems, then the classical communication capacity of $U$ is the maximum asymptotic number of classical bits that can be reliably transmitted per use of $U$. Communication can be achieved simultaneously in both directions, with possible tradeoffs. Free local resources as stated in Section I-E and shared classical randomness are always allowed.

In the context of classical communication, the most important auxiliary resource is free entanglement. Communication is called "assisted/unassisted" when this resource is/is not available.

The most general protocol with $t$ uses of $U$ can be represented as

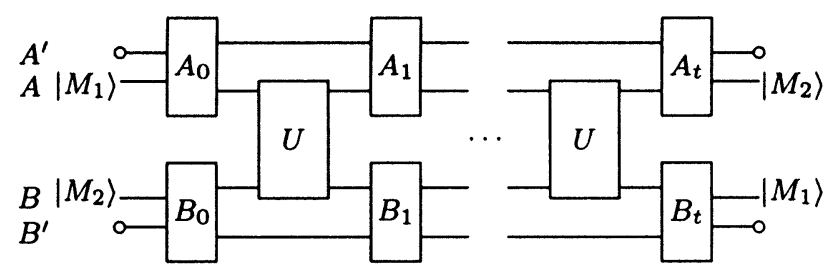

In (16), $A$ and $B$ label the systems acted on by $U$ during the protocol and the systems carrying the classical messages before and after the protocol, while $A^{\prime}$ and $B^{\prime}$ label the rest of Alice and Bob's systems. The dimensions of $A$ and $B$ are converted to $d$ by the initial operation $A_{0} \otimes B_{0}$ and are further converted by the final operation $A_{t} \otimes B_{t}$. The freedom to apply $U$ to any register is included as swap operations in $A_{i}$ and $B_{i}$. Without loss of generality, the local operations $A_{j}, B_{j}$ can be assumed unitary, 
since measurements can be deferred until the end of the protocol. In fact, no measurements are needed, except for the final readout of the transmitted messages. In the unassisted case, the initial ancilla state $\left|\psi_{\text {anc }}\right\rangle_{A^{\prime} B^{\prime}}$ can be taken to be $|0 r\rangle_{A^{\prime}}|0 r\rangle_{B^{\prime}}$ with random $r$, which can generate an arbitrary separable state. In the assisted case, $\left|\psi_{\text {anc }}\right\rangle_{A^{\prime} B^{\prime}}$ can be taken to be the maximally entangled state, which can generate an arbitrary entangled state. In both cases, the preparation of special ancillary states can be done with negligible costs. ${ }^{6}$

Each protocol allows a certain amount of forward and backward classical communication, giving a pair of achievable rates $\left(R_{\rightarrow}, R_{\leftarrow}\right)$ for the gate.

Definition 2: A pair of rates $\left(R_{\rightarrow}, R_{\leftarrow}\right)$ is said to be achievable by a gate $U$ if it is possible to intersperse $t$ uses of $U$ with local unitaries $A_{j} \otimes B_{j}$, such that an $n_{1}$-bit message $M_{1}$ from Alice to Bob and an $n_{2}$-bit message $M_{2}$ from Bob to Alice are communicated with high fidelity, and $R_{\rightarrow} \geq n_{1} / t, R_{\leftarrow} \geq n_{2} / t$. Mathematically, $\left(R_{\rightarrow}, R_{\leftarrow}\right)$ is achievable if

$\forall \epsilon>0, \exists t, \exists n_{1} \geq t R_{\rightarrow}, \exists n_{2} \geq t R_{\leftarrow}, \exists\left\{A_{j} \otimes B_{j}\right\}_{j=0}^{t}$, s.t. $\forall M_{1} \in\{0,1\}^{n_{1}}, \forall M_{2} \in\{0,1\}^{n_{2}}$,

$$
\begin{aligned}
\left|\psi_{\text {out }}\right\rangle=\left(A_{t} \otimes B_{t}\right) U\left(A_{t-1} \otimes B_{t-1}\right) U & \\
& \cdots U\left(A_{0} \otimes B_{0}\right)\left(\left|M_{1}\right\rangle_{A}\left|M_{2}\right\rangle_{B}\left|\psi_{\text {anc }}\right\rangle_{A^{\prime} B^{\prime}}\right)
\end{aligned}
$$

and $F\left(\left|M_{2}\right\rangle_{A} \otimes\left|M_{1}\right\rangle_{B}, \operatorname{tr}_{A^{\prime} B^{\prime}}\left|\psi_{\text {out }}\right\rangle\left\langle\psi_{\text {out }}\right|\right)>1-\epsilon$.

In Definition 2, the fidelity $F$ between two states $|\psi\rangle\langle\psi|$ and $\rho$ is given by $\langle\psi|\rho| \psi\rangle$ (this is a simplified expression when one of the states is pure).

We first discuss unassisted capacities, and the assisted capacities are defined in exactly the same way. Each gate $U$ defines a region of achievable unassisted rate-pairs $\left(R_{\rightarrow}, R_{\leftarrow}\right)$. The region is convex by using mixed strategies. Furthermore, if $\left(R_{\rightarrow}, R_{\leftarrow}\right)$ is achievable, so is any $\left(R_{\rightarrow}^{\prime}, R_{\leftarrow}^{\prime}\right)$ where $R_{\rightarrow}^{\prime} \leq$ $R_{\rightarrow}$ and $R_{\leftarrow}^{\prime} \leq R_{\leftarrow}$. In particular, the boundary of the achievable region never has positive slope (see Fig. 1). Thus, the forward and backward capacities can always be achieved at the boundary points, and can be defined, respectively, as

$$
\begin{aligned}
& C_{\rightarrow, U}=\sup \{R:(R, 0) \text { is achievable by } U\} \\
& C_{\leftarrow, U}=\sup \{R:(0, R) \text { is achievable by } U\} .
\end{aligned}
$$

We can also define various bidirectional capacities, for example, the duplex and the total capacities

$$
\begin{aligned}
C_{\leftrightarrow, U} & =\sup \{R:(R, R) \text { is achievable by } U\} \\
C_{+}, U & =\sup \left\{R_{\leftarrow}+R_{\rightarrow}:\left(R_{\leftarrow}, R_{\rightarrow}\right) \text { is achievable by } U\right\} .
\end{aligned}
$$

We omit the subscript $U$ when the notation is too cumbersome. Fig. 1 is a schematic diagram for the achievable region and the definitions of the various capacities. We present all the known

\footnotetext{
${ }^{6}$ In the unassisted case, free shared randomness is sufficient to create any separable state. In the assisted case, free entanglement, together with some classical communication, is sufficient to create any state. We now show that the require amount of classical communication can made negligible. Consider any protocol for sending $n c$ bits using maximally entangled states and some special ancilla $\rho_{n}$ and $n$ uses of $U$ ( $n$ is fixed but is chosen to be sufficiently large to make $c$ close to the capacity of interest). Since we are concerned with asymptotic capacities, we can consider repeating the $n$-use protocol $k$ times and focus on creating $k$ copies of $\rho_{n}$. A method is reported in [37] using $o(k)$ bits of classical communication [with a constant overhead $f(n)$ that depends on $n$ ]. Thus, the overall $k n$-use protocol transmits $k n c$ bits, requires no special ancillary states and consumes an additional $f(n) o(k)$ cbits which are negligible for fixed $n$ and sufficiently large $k$.
}

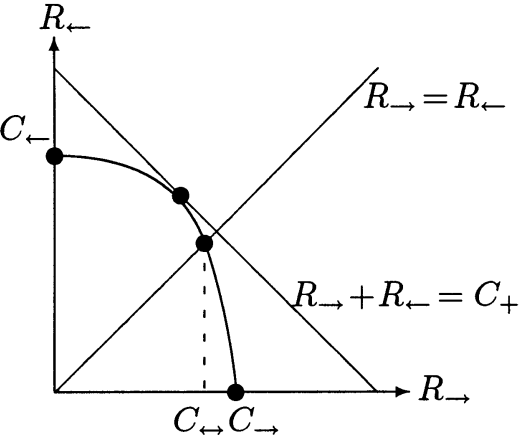

Fig. 1. Schematic diagram for the achievable region and the definitions of the various capacities. For each point $\left(R_{\leftarrow}, R_{\rightarrow}\right)$ enclosed by the curve, there is a protocol that, respectively, communicates $R_{\leftarrow}$ and $R_{\rightarrow}$ bits per use of the gate $U$ in the forward and backward directions. The one-way capacities $C_{\leftarrow}$ and $C_{\rightarrow}$ are given by the intercepts with the coordinate axes. The duplex capacity $C_{\hookrightarrow} \overrightarrow{\text { is }}$ given by the intersection with the line $R_{\leftarrow}=R_{\rightarrow}$ while the total capacity $C_{+}$ is given by the intersection with the tangent $R_{\leftarrow}+R_{\rightarrow}=C_{+}$.

properties and intentionally show the features that are not ruled out, such as the asymmetry of the region, and the nonzero curvature of the boundary.

There are much simpler examples-the unassisted achievable region for CNOT and SWAP are similar triangles with vertices $\{(0,0),(0,1),(1,0)\}$ and $\{(0,0),(0,2),(2,0)\}$, respectively, (see Section VIII).

In general, little is known about the unassisted achievable region of $\left(R_{\rightarrow}, R_{\leftarrow}\right)$ besides the convexity and the monotonicity of its boundary. The most perplexing question is perhaps whether the region has reflective symmetry about $R_{\rightarrow}=R_{\leftarrow}$, which implies $C_{\rightarrow}=C_{\leftarrow}$ and $C_{+}=2 C_{\leftrightarrow}$. References [7], [8] show that any 2-qubit gate or Hamiltonian is locally equivalent to one with Alice and Bob interchanged, so that the achievable region is indeed symmetric. This implies the conjecture in [3] that the one-shot forward and backward unassisted capacities are equal. In higher dimensions, [39] shows that there are Hamiltonians (and so unitary gates) that are intrinsically asymmetric. However, it remains open whether the achievable rate pairs are symmetric, or more weakly, whether $C_{\rightarrow}=C_{\leftarrow}$ or $C_{+}=2 C_{\leftrightarrow}$.

Assisted capacities $C_{\rightarrow}^{E}, U, C_{\leftarrow, U}^{E}, C_{\leftrightarrow}^{E}, U, C_{+, U}^{E}$ can be defined in exactly the same manner, now the ancilla $\left|\psi_{\text {anc }}\right\rangle_{A^{\prime} B^{\prime}}$ is maximally entangled instead of being $|0 r\rangle_{A^{\prime}}|0 r\rangle_{B^{\prime}}$ in the definition of the achievable rate pairs in (17) (see footnote 6). The properties and open questions of the achievable region are also the same as those in the unassisted case. Two simple examples are the assisted achievable regions for CNOT and SWAP, they are similar squares with vertices $\{(0,0),(0,1),(1,0),(1,1)\}$ and $\{(0,0),(0,2),(2,0),(2,2)\}$, respectively (see Section VIII).

Entanglement assistance greatly simplifies the analysis of the classical capacities $C_{E}$ of the usual (unidirectional) quantum channels. ${ }^{7}$ An expression for $C_{E}$ has been found and proved to be strongly additive [12], [13]. The study of $C_{E}$ also provides useful upper bounds for the unassisted capacities and insights to the classification of channels [40]. In the next section, we

\footnotetext{
${ }^{7}$ Note that the resource label $E$ appears as a subscript of the capacity for a unidirectional channel (following [12], [13]), but as a superscript for a bidirectional channel (following Section II-B).
} 
derive a simple expression for $C_{\rightarrow}^{E}, U$ and $C_{\leftarrow}^{E}, U$, the one-way (forward or backward) entanglement-assisted capacity of any bidirectional channel. Surprisingly, this capacity is also strongly additive, as in the unidirectional case!

Comparison of the two problems of generating entanglement and classical communication will be given in Section V, and the two resulting capacities are related in Section VI.

\section{Entanglement-Assisted One-Way Classical CAPACITY}

\section{A. Preliminaries and Definitions}

In this section, we derive expressions for $C_{\rightarrow, U}^{E}$ and $C_{\leftarrow, U}^{E}$, as defined in (17) with $\left|\psi_{\text {anc }}\right\rangle$ being a maximally entangled state. Without loss of generality, we focus on $C_{\rightarrow}^{E}, U$. It can be evaluated using the general framework of one-way classical communication with quantum resources [41], [10], [11]. In this framework, suppose classical messages $i$, occurring with probabilities $p_{i}$, are encoded in the "signal states" $\eta_{i}$ received by Bob, forming an ensemble $\mathcal{E}=\left\{p_{i}, \eta_{i}\right\}$. The information on $i$ obtained by measuring a signal state is upper-bounded by the Holevo information $\chi$ for the ensemble $\mathcal{E}$, defined as

$$
\chi\left(\mathcal{E}=\left\{p_{i}, \eta_{i}\right\}\right) \equiv S\left(\sum_{i} p_{i} \eta_{i}\right)-\sum_{i} p_{i} S\left(\eta_{i}\right) .
$$

The Holevo-Schumacher-Westmoreland (HSW) theorem states that this amount of mutual information per signal state is achievable given the ability to transmit an asymptotically large number of signal states. (See [10], [11], and [14, Ch. 12.3.2].)

We will see that the optimal methods to generate EPR pairs (see Sections II-D-II-E) and entanglement-assisted classical communication have many similarities. The respective goals are to maximize the increase in entanglement and the Holevo information. The optimal asymptotic strategies in both cases are to repeat the 1-shot protocol, with an optimal input state in the former and with an optimal input ensemble in the latter. In the case of entanglement generation, allowing the most general 1-shot optimal input with arbitrary ancillas and initial entanglement makes the 1-shot capacity equal to the asymptotic ones. Likewise, we will allow the most general 1-shot input ensemble for assisted classical communication, and will show that the resulting 1-shot capacity is equal to the asymptotic capacities by establishing a method to "replenish" the optimal input ensemble (analogous to concentration and dilution in entanglement generation).

Let $\mathcal{E}=\left\{p_{i},\left|\psi_{i}\right\rangle_{A A^{\prime} B B^{\prime}}\right\}$ be an ensemble of bipartite states. A trace-preserving operation acts on $\mathcal{E}$ by acting on each component state (preserving its probability). For example, we will write

$$
\begin{aligned}
U \mathcal{E} & =\left\{p_{i}, U_{A B}\left|\psi_{i}\right\rangle_{A A^{\prime} B B^{\prime}}\right\} \\
\operatorname{tr}_{A A^{\prime}} \mathcal{E} & =\left\{p_{i}, \operatorname{tr}_{A A^{\prime}}\left|\psi_{i}\right\rangle\left\langle\left.\psi_{i}\right|_{A A^{\prime} B B^{\prime}}\right\}\right.
\end{aligned}
$$

and

$$
\operatorname{tr}_{A A^{\prime}} U \mathcal{E}=\left\{p_{i}, \operatorname{tr}_{A A^{\prime}} U_{A B}\left|\psi_{i}\right\rangle\left\langle\left.\psi_{i}\right|_{A A^{\prime} B B^{\prime}} U_{A B}^{\dagger}\right\} .\right.
$$

We have the following definitions analogous to those in Section II-B.

Definition 3: The $t$-shot Holevo information capacity of $U$ is the maximum increase in Holevo information per use of $U$ due to any protocol $\mathcal{P}_{t}$ that uses $U t$ times, the auxiliary resources labeled $r$, and the local resources specified in Section I-E. There are two possible $t$-shot capacities, depending on the allowed input ensembles:

1) when the input ensemble $\mathcal{E}_{0}$ is restricted to satisfy $\chi\left(\operatorname{tr}_{A A^{\prime}} \mathcal{E}_{0}\right)=0$

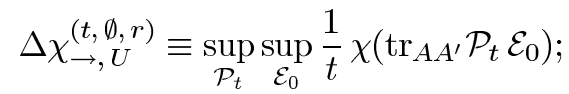

2) when the input ensemble $\mathcal{E}$ is unrestricted

$$
\Delta \chi_{\rightarrow, U}^{(t, *, r)} \equiv \sup _{\mathcal{P}_{t}} \sup _{\mathcal{E}} \frac{1}{t}\left[\chi\left(\operatorname{tr}_{A A^{\prime}} \mathcal{P}_{t} \mathcal{E}\right)-\chi\left(\operatorname{tr}_{A A^{\prime}} \mathcal{E}\right)\right] \text {. }
$$

Since we always assume free entanglement as an auxiliary resource, and we always focus on forward capacity, we omit $r=$ $E$ and $\rightarrow$ in the above notation

$$
\Delta \chi_{U}^{(t, \emptyset)} \equiv \Delta \chi_{\rightarrow, U}^{(t, \emptyset, E)} \quad \text { and } \quad \Delta \chi_{U}^{(t, *)} \equiv \Delta \chi_{\rightarrow, U}^{(t, *, E)}
$$

We have

$$
C_{\rightarrow, U}^{E}=\sup _{t} \Delta \chi_{U}^{(t, \emptyset)}
$$

Note that it is unnecessary to consider mixed-state ensembles in (19) and (20) — we can replace a mixed state $\rho_{A A^{\prime} B B^{\prime}}$ by its

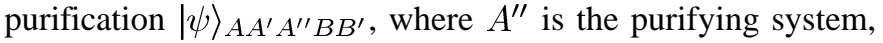
without affecting neither $\operatorname{tr}_{A A^{\prime} A^{\prime \prime}} \mathcal{E}$ nor $\operatorname{tr}_{A A^{\prime} A^{\prime \prime}} \mathcal{P}_{t} \mathcal{E}$.

In the next two subsections, we will prove $C_{\rightarrow, U}^{E}=\Delta \chi_{U}^{(1, *)}$. We first prove that $C_{\rightarrow}^{E}, U \leq \Delta \chi_{U}^{(1, *)}$, and then we describe a protocol to achieve the upper bound, thereby proving additivity and providing an optimal asymptotic strategy.

\section{B. An Additive Upper Bound}

We first prove an analog of Lemma 1.

Lemma 5: $C_{\rightarrow, U}^{E} \leq 2 \log d$ and $C_{\leftarrow}^{E}, U \leq 2 \log d$.

Proof: Consider a $t$-use protocol. Replace each use of $U$ by double teleportation (see Lemma 1). If the original protocol consumes and produces $C_{\text {in }}$ and $C_{\text {out }}$ bits of forward communication, the modified protocol consumes and produces $C_{\text {in }}+2 t \log d$ and $C_{\text {out }}$ bits of forward communication. By causality [42] of the modified protocol, $2 t \log d \geq C_{\text {out }}-C_{\text {in }}$. Hence, $C_{\rightarrow, U}^{E} \leq 2 \log d$. Similarly, $C_{\leftarrow, U}^{E} \leq 2 \log d$. (Note that the above proof is stronger than we need, since we have allowed $C_{\text {in }} \neq 0$.)

Consider the best 1-shot protocol to increase the Holevo information. Since local operations do not increase mutual information, the optimal 1-shot protocol is to just apply $U$, as in (6). Thus,

$$
\Delta \chi_{U}^{(1, *)}=\sup _{\mathcal{E}}\left[\chi\left(\operatorname{tr}_{A A^{\prime}} U \mathcal{E}\right)-\chi\left(\operatorname{tr}_{A A^{\prime}} \mathcal{E}\right)\right]
$$

where the supremum is over the most general bipartite pure state ensemble $\mathcal{E}=\left\{p_{i},\left|\psi_{i}\right\rangle_{A A^{\prime} B B^{\prime}}\right\}$.

We now consider the asymptotic problem. Using the same idea that proves Theorem 1 (fisst half), we obtain the following analog.

Theorem 2 (First Half): $\Delta \chi_{U}^{(t, *)} \leq \Delta \chi_{U}^{(1, *)}$.

Proof: Consider the most general protocol $\mathcal{P}_{t}$ with $t$ uses of $U$ (such as depicted in (16)). Let $\mathcal{E}$ be an arbitrary bipartite input ensemble. Then, the total increase in $\chi$ is upper-bounded 
by the sum of the stepwise increases. Since local operations cannot increase $\chi$, and the increase in $\chi$ by each use of $U$ is bounded by (23)

$$
\chi\left(\operatorname{tr}_{A A^{\prime}} \mathcal{P}_{t} \mathcal{E}\right)-\chi\left(\operatorname{tr}_{A A^{\prime}} \mathcal{E}\right) \leq t \Delta \chi_{U}^{(1, *)}
$$

from which the theorem is immediate.

It follows from (24) and (22) that $C_{\rightarrow}^{E} \leq \Delta \chi_{U}^{(1, *)}$.

\section{Protocol to Achieve the Upper Bound of $C_{\rightarrow}^{E}, U$}

In optimal asymptotic entanglement generation, the following basic cycle is repeated:

1) convert EPR pairs into $n$ copies of the optimal input state;

2) apply the gate to each;

3) convert the $n$ copies of optimal output state into EPR pairs.

More EPR pairs are obtained in 3) than used in 1) - as excess entanglement generated.

In entanglement-assisted classical communication, we want a similar basic cycle:

1) convert classical communication to create $n$ states drawn from the optimal input ensemble;

2) apply the gate to each state;

3) convert the states from the optimal output ensemble into classical communication.

Step 1) is called remote state preparation (RSP) [33], [43], [44], a procedure whereby Alice helps Bob to construct quantum states of her choice in his laboratory using entanglement and classical communication. The most general procedure in RSP is that Alice performs a measurement on her half of the shared entangled state, sends the outcome to Bob, who conditioned on the outcome operates on his half of the shared entangled state to complete the RSP. For our problem, it is known [45], [46] how to approximately prepare $n$ pure bipartite states from an ensemble $\mathcal{E}$ with free entanglement and $n \chi\left(\operatorname{tr}_{A A^{\prime}} \mathcal{E}\right)+o(n)$ bits of classical communication. Step 3) follows from the HSW theorem: Alice can communicate $\approx n \chi\left(\operatorname{tr}_{A A^{\prime}} U \mathcal{E}\right)-o(n)$ bits to Bob reliably if she can prepare $n$ states in the output ensemble $U \mathcal{E}$. Just like the case of generating entanglement, $n$ is chosen large enough to ensure the efficiency of steps 1) and 3).

When describing and analyzing the protocol, we loosely call $\mathcal{E}$ the optimal ensemble achieving the supremum in (23). For arbitrarily small $\epsilon, \mathcal{E}$ is chosen so that

$$
\chi\left(\operatorname{tr}_{A A^{\prime}} U \mathcal{E}\right)-\chi\left(\operatorname{tr}_{A A^{\prime}} \mathcal{E}\right) \geq \Delta \chi_{U}^{(1, *)}-\epsilon .
$$

Since it is obvious how $\epsilon$ enters the following analysis, and the analysis is independent of the choice of $\epsilon$ and $\mathcal{E}, \epsilon$ is omitted for simplicity.

Protocol That Achieves $C_{\rightarrow}^{E}, U=\Delta \chi_{U}^{(1, *)}$ : Let $\mathcal{E}$ be the optimal ensemble. If Alice is given $n \chi\left(\operatorname{tr}_{A A^{\prime}} \mathcal{E}\right)$ bits of classical communication as an initial resource, she can transmit $k$ messages $M_{i=1, \ldots, k}$ each of length $n \Delta \chi_{U}^{(1, *)}$ (a total of $n k \Delta \chi_{U}^{(1, *)}$ bits) with $n k$ uses of $U$ as follows.

- Alice's Preprocessing: Alice determines $k$ messages $N_{i}$ each of $n \chi\left(\operatorname{tr}_{A A^{\prime}} U \mathcal{E}\right)$ bits. Each $N_{i}$ has two parts: the message $M_{i}$ of length $n \Delta \chi_{U}^{(1, *)}$, and an RSP instruction $R_{i}$ of length $n \chi\left(\operatorname{tr}_{A A^{\prime}} \mathcal{E}\right)$ for Bob to create a state $\left|\phi_{i+1}\right\rangle \in \mathcal{E}^{\otimes n}$ such that

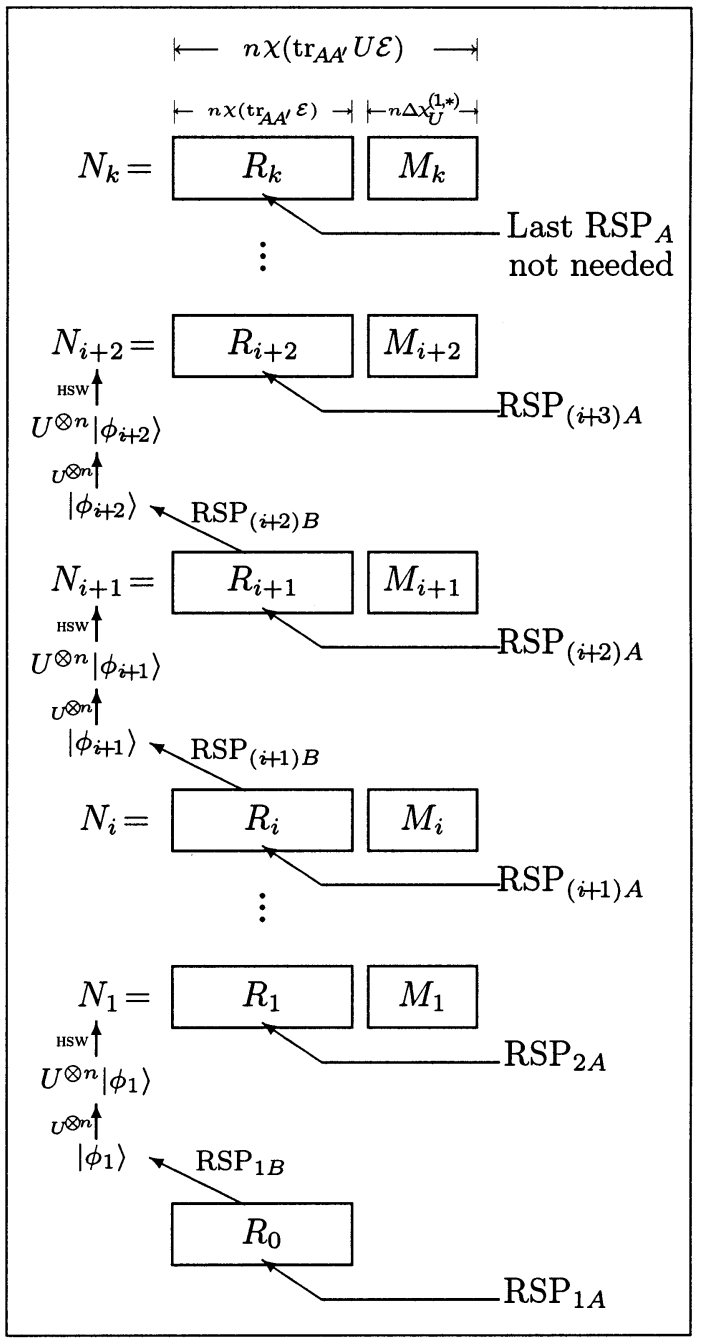

Fig. 2. A protocol that achieves $C_{\rightarrow}^{E}, U=\Delta \chi_{U}^{(1, *)}$. Time increases along the upwards direction. In each step, Alice performs a measurement $\operatorname{RSP}_{i A}$ on her half of the shared entanglement (free) to obtain the instruction $R_{i-1}$ for Bob to prepare $\left|\phi_{i}\right\rangle$. $\operatorname{RSP}_{i B}$ denotes Bob's conditional operation to complete the preparation of $\left|\phi_{i}\right\rangle$. Then $n$ uses of the gate $U$ are applied to obtain the state $U \otimes n\left|\phi_{i}\right\rangle$, which encodes the message $N_{i}$. Bob applies the appropriate decoding procedure (denoted as "HSW") to extract $N_{i}$, which has two parts, the RSP instruction $R_{i}$ for the next step and some message $M_{i}$ that is to be communicated by the protocol. Part of the communication generated in each step is used in the next.

$U^{\otimes n}\left|\phi_{i+1}\right\rangle \in(U \mathcal{E})^{\otimes n}$ encodes $N_{i+1}$ (by the HSW theorem). In order to generate $R_{i}$ for $N_{i}$, Alice needs to determine $\left|\phi_{i+1}\right\rangle$ and to perform her measurement for the RSP of $\left|\phi_{i+1}\right\rangle$. This, in turn, requires knowledge of $N_{i+1}$. So Alice first computes the last message $N_{k}$ (in which $M_{k}$ is known and $R_{k}$ is irrelevant), classically calculates $\left|\phi_{k}\right\rangle$, performs measurement for the RSP of $\left|\phi_{k}\right\rangle$ to find $R_{k-1}$ in $N_{k-1}$, works her way backward through $N_{k-1}, \ldots, N_{1}$, determining $\left|\phi_{i}\right\rangle$ from $N_{i}$ and performing measurement for RSP for $\left|\phi_{i}\right\rangle$ for decreasing $i$.

- Quantum Protocol: Alice uses the given initial classical communication to create $\left|\phi_{1}\right\rangle$, which she shares with Bob. Then $U^{\otimes n}$ is applied to convert it to $U^{\otimes n}\left|\phi_{1}\right\rangle$, Bob reads off the message $N_{1}$, which consists of $R_{1}$ to instruct him to do RSP for $\left|\phi_{2}\right\rangle$, and so on.

The protocol is summarized in Fig. 2. 
The initial amount of classical communication can be created by Alice and Bob using $c n$ uses of $U$ inefficiently, for some constant $c .{ }^{8}$ The communication rate is

$$
\frac{k n \Delta \chi_{U}^{(1, *)}}{c n+n k} \stackrel{k \rightarrow \infty}{\longrightarrow} \Delta \chi_{U}^{(1, *)}
$$

We have not yet discussed small inaccuracies and inefficiencies in the protocol. The asymptotic correctness of this protocol comes from the asymptotic reliability of its component pieces: RSP and the HSW theorem. However, since errors and inefficiencies accumulate over many rounds, we need to choose the rates of increase of $n$ and $k$ slightly more carefully.

Suppose that preparing a member of $\mathcal{E}^{\otimes n}$ with RSP requires $n\left(\chi\left(\operatorname{tr}_{A A^{\prime}} \mathcal{E}\right)+\delta_{n}^{\mathrm{RSP}}\right)$ bits of communication and has error $\epsilon_{n}^{\mathrm{RSP}}$, where $\delta_{n}^{\mathrm{RSP}}, \epsilon_{n}^{\mathrm{RSP}} \rightarrow 0$ as $n \rightarrow \infty$. Similarly, a state in $(U \mathcal{E})^{\otimes n}$ provides $n\left(\chi\left(\operatorname{tr}_{A A^{\prime}} U \mathcal{E}\right)-\delta_{n}^{\mathrm{HSW}}\right)$ bits of information with error $\epsilon_{n}^{\mathrm{HSW}}$, where, again, $\delta_{n}^{\mathrm{HSW}}, \epsilon_{n}^{\mathrm{HSW}} \rightarrow 0$ as $n \rightarrow \infty$. Combining these into $\delta_{n}=\delta_{n}^{\mathrm{RSP}}+\delta_{n}^{\mathrm{HSW}}$ and $\epsilon_{n}=\epsilon_{n}^{\mathrm{RSP}}+\epsilon_{n}^{\mathrm{HSW}}$, we find that the communication rate is

$$
\begin{aligned}
\frac{k n\left(\Delta \chi_{U}^{(1, *)}-\delta_{n}\right)}{c n+n k} & =\left(\Delta \chi_{U}^{(1, *)}-\delta_{n}\right)\left(1-\frac{c}{k}+O\left(k^{-2}\right)\right) \\
& \stackrel{n, k \rightarrow \infty}{\longrightarrow} \Delta \chi_{U}^{(1, *)}
\end{aligned}
$$

and the total error is $k \epsilon_{n}$. This vanishes if one chooses $k$ first, and then chooses $n$ such that $\epsilon_{n} k$ is small ( $n$ thus depends on $k$ ).

We summarize the order of the limits. First, choose the optimal ensemble $\mathcal{E}$ to approximate $\Delta \chi_{U}^{(1, *)}$. Second, choose $k$ large to make $c / k$ negligible (to overcome the initial cost). Finally, choose $n$ large to make both of $k \epsilon_{n}$ and $\delta_{n}$ vanish. As this protocol does not require initial mutual information, we have the following.

Theorem 2 (Second Half): $\lim _{t \rightarrow \infty} \Delta \chi_{U}^{(t, \emptyset)} \geq \Delta \chi_{U}^{(1, *)}$.

Putting the two halves together gives:

Theorem 2:

$$
\begin{aligned}
C_{\rightarrow}^{E} & =\lim _{t \rightarrow \infty} \Delta \chi_{U}^{(t, \emptyset)}=\Delta \chi_{U}^{(1, *)} \\
& =\sup _{\mathcal{E}}\left[\chi\left(\operatorname{tr}_{A A^{\prime}} U \mathcal{E}\right)-\chi\left(\operatorname{tr}_{A A^{\prime}} \mathcal{E}\right)\right]
\end{aligned}
$$

Thus, initial mutual information does not increase the asymptotic capacity, analogous to entanglement generation. Finally, we generalize Theorem 2 to prove strong additivity.

Theorem 2S: The classical communication achievable by $n_{i}$ uses of $U_{i}$ is asymptotically $\sum_{i} n_{i} \Delta \chi_{U_{i}}^{(1, *)}$.

Proof: The argument that proves Theorem 2 (first half) can be applied to prove that the amount of communication generated is no more than $\sum_{i} n_{i} \Delta \chi_{U_{i}}^{(1, *)}$, which is achieved by applying the optimal protocol for each $U_{i}$ separately.

\section{Additivity}

We conclude this section with two observations about additivity.

${ }^{8}$ Section VI shows that any nonlocal gate $U$ has nonzero communication capacities in both directions.
- We emphasize that in Theorem 2 (first half), the Holevo bound is applied to the output of a general protocol $\mathcal{P}_{t}$ with possibly entangled inputs to different uses of $U$. Thus, the one-way entanglement-assisted capacity for unitary bidirectional channels is strongly additive independent of whether the Holevo information $\chi$ is additive or superadditive.

- In the optimal asymptotic protocol, the $n$ copies of $U$ are applied to $n$ states each chosen from the optimal input ensemble. Thus, entangling the inputs to different uses of $U$ does not improve $C_{\rightarrow}^{E}, U$.

\section{DISCUSSION}

Despite the many similarities between generating entanglement and entanglement-assisted classical communication, there is an important difference. Communication cannot be stored and be used later. In particular, Alice needs to work backward in our optimal entanglement-assisted communication protocol, so that the classical messages need to be known at the beginning of the protocol to share the initial cost. In contrast, entanglement can be stored. The optimal entanglement generation protocol can be stopped and resumed at arbitrary times.

We can generalize the first half of Theorems 1 and 2 to any other quantity which is monotonic under the given resources, as long as a sufficiently general input (e.g., state or ensemble) is allowed for the 1-shot capacity. In particular, the input should possess all the properties the output may possess. If, in addition, the quantity is weakly additive or subadditive on the optimal input and weakly additive or superadditive on the optimal output, repeating the optimal 1-shot protocol allows the upper bound to be attained asymptotically, and additivity holds.

We end this section with a discussion on the parallel versus sequential applications of bidirectional channels in a protocol. Note that there is no such distinction for unidirectional channels (in the absence of back channels), as the output state of a given application of the channel is with the receiver and can never be used as an input for later uses. For bidirectional channels, there are sequential schemes that cannot be made parallel. For example, the protocol for entanglement-assisted one-way classical communication in Section IV-C cannot be made parallel. Sequential schemes are always at least as powerful as parallel ones. The opposite is true in the asymptotic regime, in which case any capacity of $U^{\otimes n}$ (i.e., one must apply $n$ copies of $U$ in parallel) is equal to $n$ times the capacity of $U$. The proof is simple-let $\mathcal{P}_{t}$ be any protocol that uses $U$ sequentially. A particular $t$-use protocol for $U^{\otimes n}$ is to run $n$ copies of $\mathcal{P}_{t}$ in parallel. Thus, the $t$-shot capacity of $U^{\otimes n}$ is no worse than $n$ times that of $U$, and equality holds.

\section{OTHER GENERAL BOUNDS}

We have proved a few simple general bounds:

$$
\begin{gathered}
E_{U}^{(*, c c)} \leq \log \operatorname{Sch}(U) \leq 2 \log d \\
C_{\rightarrow, U}^{E}, C_{\leftarrow, U}^{E} \leq 2 \log d .
\end{gathered}
$$

We now derive other general bounds that hold for all $U$. We focus on the entropy of entanglement $E_{e}$, and on the two ca- 
pacities $E_{e, U}^{(1, \emptyset)}$ and $E_{e, U}^{(\emptyset)}\left(=E_{e, U}^{(1, *)}\right)$ since the latter is equal to many entanglement capacities of our interest (see Section II-F).

Bound 1: $U$ is nonlocal $\Leftrightarrow E_{e, U}^{(1, \emptyset)}>0 \Leftrightarrow C_{\rightarrow, U}>0 \Leftrightarrow$ $C_{\leftarrow, U}>0$.

Proof: The first equivalence follows from Corollary 3.5 in Section II-D. Let $E_{0}>0$ be the amount of entanglement created by applying $U$ to $\left|\Phi_{d}\right\rangle_{A A^{\prime}}\left|\Phi_{d}\right\rangle_{B B^{\prime}}$.

Alice can send a noisy bit to Bob with the following $t$-use protocol. Bob inputs $\left|\Phi_{d}\right\rangle_{B B^{\prime}}^{\otimes t}$ to all $t$ uses of $U$. To send " 0 " Alice inputs $\left|\Phi_{d}\right\rangle_{A A^{\prime}}^{\otimes t}$ to share $t E_{0}$ ebit with Bob. To send " 1 ," Alice inputs $|0\rangle_{A}$ to the first use of $U$, takes the output and uses it as the input to the second use, and so on, so that their final entanglement is no more than $\log d$. Thus, different messages from Alice result in a very different amount of entanglement at the end of the protocol. Using Fannes' inequality [47], [48]

$$
t E_{0}-\log d \leq t \log d \operatorname{tr}\left|\rho_{0}-\rho_{1}\right|+\frac{1}{e}
$$

where $\rho_{i}$ are the reduced density matrices of Bob when Alice sends $i$. For any $\epsilon>0, \exists t$ such that $E_{0}-\epsilon \leq \log d \operatorname{tr}\left|\rho_{0}-\rho_{1}\right|$ and Bob can distinguish $\rho_{0}$ from $\rho_{1}$ with nonzero advantage. It means that the $t$-use protocol then simulates a noisy classical channel with nonzero capacity and $C_{\rightarrow, U}>0$. Obviously, $C_{\rightarrow, U}>0$ implies $U$ is nonlocal. Similarly, $C_{\rightarrow, U}>0 \Leftrightarrow U$ is nonlocal.

Bound 2: $E_{U}^{(\emptyset)} \geq C_{+, U}$.

Proof: Suppose a $t$-use protocol $\mathcal{P}_{t}$ transmits $n_{a}$ bits from Alice to Bob and $n_{b}$ bits from Bob to Alice with fidelity $1-\epsilon$. Recall from Section III that $\mathcal{P}_{t}$ can be assumed unitary with the ancillas starting in the state $|0 r\rangle_{A^{\prime}}|0 r\rangle_{B^{\prime}}$, where $r$ is a shared random variable. Let $|x\rangle_{A}|y\rangle_{B}$ carry the messages to be communicated, where $x$ and $y$ are $n_{a}$ - and $n_{b}$-bit strings. Then, by definition (17), the state change is given by

$$
\begin{aligned}
& |x\rangle_{A}|y\rangle_{B}|0 r\rangle_{A^{\prime}}|0 r\rangle_{B^{\prime}} \stackrel{\mathcal{P}_{t}}{\longrightarrow}\left|\eta_{x y}\right\rangle \quad \text { s.t. } \\
& \forall_{x, y} F\left(|y\rangle_{A}|x\rangle_{B}, \operatorname{tr}_{A^{\prime} B^{\prime}}\left|\eta_{x y}\right\rangle\left\langle\left.\eta_{x y}\right|_{A B A^{\prime} B^{\prime}}\right)\right. \\
& \quad=1-\epsilon_{x y}>1-\epsilon .
\end{aligned}
$$

By Uhlmann's theorem [49], there are normalized states $\left|c_{x y}\right\rangle_{A^{\prime} B^{\prime}}$ and $\left|e_{x y}\right\rangle_{A B A^{\prime} B^{\prime}}$ such that

$$
\left|\eta_{x y}\right\rangle=\sqrt{1-\epsilon_{x y}}|y\rangle_{A}|x\rangle_{B}\left|c_{x y}\right\rangle_{A^{\prime} B^{\prime}}+\sqrt{\epsilon_{x y}}\left|e_{x y}\right\rangle_{A B A^{\prime} B^{\prime}}
$$

and $\operatorname{tr}_{A^{\prime} B^{\prime}}\left|e_{x y}\right\rangle\left\langle\left. e_{x y}\right|_{A B A^{\prime} B^{\prime}}\right.$ has support orthogonal to the span of $|y\rangle_{A}|x\rangle_{B}$.

To prove $E_{U}^{(\emptyset)} \geq C_{+}, U$, we simply change the inputs to the protocol so that it creates entanglement. Alice's input system $A$ is now in a maximally entangled state with another ancilla $A^{\prime \prime}$, each with $2^{n_{a}}$ dimensions, and similarly for Bob. Thus, the input state is given by

$$
2^{-\left(n_{a}+n_{b}\right) / 2} \sum_{x y}|x\rangle_{A}|x\rangle_{A^{\prime \prime}}|y\rangle_{B}|y\rangle_{B^{\prime \prime}}|0 r\rangle_{A^{\prime}}|0 r\rangle_{B^{\prime}}
$$

where $x$ and $y$ are summed over their possible values. The output is given by

$$
\left|\eta^{(\epsilon)}\right\rangle=2^{-\left(n_{a}+n_{b}\right) / 2} \sum_{x y}\left|\eta_{x y}\right\rangle
$$

$$
\begin{aligned}
=2^{-\left(n_{a}+n_{b}\right) / 2} \sum_{x y}[ & \sqrt{1-\epsilon_{x y}}|y\rangle_{A}|x\rangle_{B}\left|c_{x y}\right\rangle_{A^{\prime} B^{\prime}} \\
& \left.+\sqrt{\epsilon_{x y}}\left|e_{x y}\right\rangle_{A B A^{\prime} B^{\prime}}\right]|x\rangle_{A^{\prime \prime}}|y\rangle_{B^{\prime \prime}} .
\end{aligned}
$$

To calculate $E\left(\left|\eta^{(\epsilon)}\right\rangle\right)$, we first calculate $E\left(\left|\eta^{(0)}\right\rangle\right)$ for

$$
\left|\eta^{(0)}\right\rangle=2^{-\left(n_{a}+n_{b}\right) / 2} \sum_{x y}|y\rangle_{A}|x\rangle_{A^{\prime \prime}}|x\rangle_{B}|y\rangle_{B^{\prime \prime}}\left|c_{x y}\right\rangle_{A^{\prime} B^{\prime}}
$$

$E\left(\left|\eta^{(0)}\right\rangle\right)$ is simply the entropy of Alice's reduced density matrix, which can be found by the "Joint Entropy Theorem" (in $[14$, eq. (1.58)])

$$
\begin{aligned}
& S\left(\operatorname{tr}_{B B^{\prime} B^{\prime \prime}}\left|\eta^{(0)}\right\rangle\left\langle\eta^{(0)}\right|\right) \\
& \quad=S\left(2^{-\left(n_{a}+n_{b}\right)} \sum_{x y}|y\rangle\left\langle\left. y\right|_{A} \mid x\right\rangle\left\langle\left. x\right|_{A^{\prime \prime}} \otimes \operatorname{tr}_{B^{\prime}} \mid c_{x y}\right\rangle\left\langle c_{x y}\right|\right) \\
& \quad=n_{a}+n_{b}+2^{-\left(n_{a}+n_{b}\right)} \sum_{x y} S\left(\operatorname{tr}_{B^{\prime}}\left|c_{x y}\right\rangle\left\langle c_{x y}\right|\right) \\
& \quad \geq n_{a}+n_{b} .
\end{aligned}
$$

We now relate $E\left(\left|\eta^{(\epsilon)}\right\rangle\right)$ to $E\left(\left|\eta^{(0)}\right\rangle\right)$. The inner product $\left\langle\eta^{(0)} \mid \eta^{(\epsilon)}\right\rangle$ can be bounded as follows:

$$
\begin{aligned}
\left\langle\eta^{(0)} \mid \eta^{(\epsilon)}\right\rangle & 2^{-\left(n_{a}+n_{b}\right)} \sum_{x y}\left\langley | _ { A } \left\langlex | _ { B } \left\langle\left. c_{x y}\right|_{A^{\prime} B^{\prime}}\right.\right.\right. \\
& {\left[\sqrt{1-\epsilon_{x y}}|y\rangle_{A}|x\rangle_{B}\left|c_{x y}\right\rangle_{A^{\prime} B^{\prime}}+\sqrt{\epsilon_{x y}}\left|e_{x y}\right\rangle_{A B A^{\prime} B^{\prime}}\right] } \\
= & 2^{-\left(n_{a}+n_{b}\right)} \sum_{x y} \sqrt{1-\epsilon_{x y}} \\
\geq & \sqrt{1-\epsilon}
\end{aligned}
$$

where we have used the orthogonality of $\left\{|x\rangle_{A^{\prime \prime}}|y\rangle_{B^{\prime \prime}}\right\}_{x y}$ to obtain the first line, and the orthogonality of $|y\rangle_{A}|x\rangle_{B}\left|c_{x y}\right\rangle_{A^{\prime} B^{\prime}}$ and $\left|e_{x y}\right\rangle_{A B A^{\prime} B^{\prime}}$ to obtain the second line. The trace distance $D$ between $\left|\eta^{(0)}\right\rangle$ and $\left|\eta^{(\epsilon)}\right\rangle$ is defined to be

$$
\left.\frac{1}{2} \operatorname{tr}|| \eta^{(0)}\right\rangle\left\langle\eta^{(0)}|-| \eta^{(\epsilon)}\right\rangle\left\langle\eta^{(\epsilon)} \|\right.
$$

and

$$
D\left(\left|\eta^{(0)}\right\rangle,\left|\eta^{(\epsilon)}\right\rangle\right)=\sqrt{1-\left\langle\eta^{(0)} \mid \eta^{(\epsilon)}\right\rangle^{2}} \leq \sqrt{\epsilon}
$$

(see [14, Sec. 9.2.3]). Using Fannes' inequality [47] or the continuity of the entropy of entanglement [48]

$$
\begin{aligned}
& \mid E\left(\left|\eta^{(0)}\right\rangle\right)-E\left(\left|\eta^{(\epsilon)}\right\rangle\right) \mid \\
& \leq \log \left(\operatorname{Sch}\left(\left|\eta^{(0)}\right\rangle\right)+\operatorname{Sch}\left(\left|\eta^{(\epsilon)}\right\rangle\right)\right) \\
& \quad \cdot 2 D\left(\left|\eta^{(0)}\right\rangle,\left|\eta^{(\epsilon)}\right\rangle\right)+1 / e \\
& \leq\left(2 t \log d+n_{a}+n_{b}\right) 2 \sqrt{\epsilon}+1 / e .
\end{aligned}
$$

We explain how (33) is obtained. First, $\operatorname{Sch}\left(\left|\eta^{(\epsilon)}\right\rangle\right) \leq d^{2 t}$ because the protocol has a product initial state and $\operatorname{Sch}(U|\psi\rangle) \leq$ $\operatorname{Sch}(U) \operatorname{Sch}(|\psi\rangle)$ [36]. We now bound $\operatorname{Sch}\left(\left|\eta^{(0)}\right\rangle\right)$. A Schmidt decomposition of $\left|\eta^{(0)}\right\rangle$ can be obtained by Schmidt decomposing each $\left|c_{x y}\right\rangle$ in (30) so that

$$
\operatorname{Sch}\left(\left|\eta^{(0)}\right\rangle\right)=\sum_{x y} \operatorname{Sch}\left(\left|c_{x y}\right\rangle\right)
$$

For each $x y, \operatorname{Sch}\left(\left|c_{x y}\right\rangle\right) \leq \operatorname{Sch}\left(\left|\eta_{x y}\right\rangle\right)$ since $\left|c_{x y}\right\rangle$ can be obtained with nonzero probability by locally measuring $\left|\eta_{x y}\right\rangle$. 
Each $\left|\eta_{x y}\right\rangle$ is obtained from a product initial state after $t$ applications of $U$, and $\operatorname{Sch}\left(\left|\eta_{x y}\right\rangle\right) \leq d^{2 t}$. Altogether

$$
\operatorname{Sch}\left(\left|\eta^{(0)}\right\rangle\right) \leq d^{2 t} 2^{n_{a}+n_{b}}
$$

and

$$
\operatorname{Sch}\left(\left|\eta^{(0)}\right\rangle\right)+\operatorname{Sch}\left(\left|\eta^{(\epsilon)}\right\rangle\right) \approx d^{2 t} 2^{n_{a}+n_{b}} .
$$

From (31) and (33) we can lower-bound the entanglement generated per use of $U$

$$
\begin{aligned}
& \frac{1}{t} E\left(\left|\eta^{(\epsilon)}\right\rangle\right) \\
& \quad \geq \frac{1}{t}\left(n_{a}+n_{b}\right)-2 \sqrt{\epsilon}\left[2 \log d+\frac{1}{t}\left(n_{a}+n_{b}\right)\right]-\frac{1}{e t} .
\end{aligned}
$$

As $t$ increases, $\epsilon$ can be made arbitrarily small and $\frac{1}{t}\left(n_{a}+\right.$ $\left.n_{b}\right) \rightarrow C_{+, U}$. Furthermore, $2 \log d+\left(n_{a}+n_{b}\right) / t \rightarrow 2 \log d+$ $C_{+, U} \leq 4 \log d$ is well bounded. The above equation then implies $E_{U}^{(\emptyset)} \geq C_{+, U}$.

Remark: In the proof above, it is crucial to bound $\operatorname{Sch}\left(\left|\eta^{(\epsilon)}\right\rangle\right)$ and $\operatorname{Sch}\left(\left|\eta^{(0)}\right\rangle\right)$ as functions of $t$, and our bound is based on having a product initial state. Furthermore, the two limits $t \rightarrow \infty$ and $\epsilon \rightarrow 0$ are dependent. Thus, one cannot assume $\epsilon \rightarrow 0$ as a separate premise in the above proof and extra care is needed in how the limits are taken.

After this paper was first posted, Berry and Sanders [50] proved that if the capacity is achievable by an exact protocol (i.e., $\epsilon=0$ ), then $C_{+, U}^{E} \leq E_{U}^{(\emptyset)}+E_{U^{\dagger}}^{(\emptyset)}$.

To adapt the proof of Bound 2 for $C_{+, U}^{E} \leq E_{U}^{(\emptyset)}+E_{U^{\dagger}}^{(\emptyset)}$ in the general case when $\epsilon>0$ will require an explicit bound on $\operatorname{Sch}\left(\left|\eta^{(\epsilon)}\right\rangle\right)$ and $\operatorname{Sch}\left(\left|\eta^{(0)}\right\rangle\right)$ and knowledge of how various inaccuracies vanish asymptotically, so as to specify how various dependent limits should be taken. So far, we do not see how this can be done.

In the following, we prove a weaker bound

$$
C_{\rightarrow, U}^{E} \leq E_{U}^{(\emptyset)}+E_{U^{\dagger}}^{(\emptyset)}, \quad \text { for } \epsilon>0
$$

by adapting the proof of Bound 2 and an idea from [50], as well as using details on the optimal protocol for achieving $C_{\rightarrow, U}^{E}$ and an improved method for RSP of bipartite pure entangled state that uses less entanglement than the method in [45].

Before we present the proof, we give an interpretation of $E_{U^{\dagger}}^{(\emptyset)}=E_{U^{\dagger}}^{(1, *)}$ as the entanglement destroying capacity of $U$

$$
\sup _{|\psi\rangle_{A A^{\prime} B B^{\prime}}}\left[E\left(|\psi\rangle_{A A^{\prime} B B^{\prime}}\right)-E\left(U_{A B}|\psi\rangle_{A A^{\prime} B B^{\prime}}\right)\right]
$$

since $U^{\dagger}$ creates as much entanglement on the input $|\psi\rangle$ as $U$ can destroy on $U^{\dagger}|\psi\rangle$. Note that to disentangle a state unitarily is a nonlocal task. We now turn to our proof.

Bound 3: $C_{\rightarrow, U}^{E} \leq E_{U}^{(\emptyset)}+E_{U^{\dagger}}^{(\emptyset)}$.

Proof: We omit details already given in the proof of Bound 2. Let $\mathcal{P}_{t}$ be a unitary protocol transmitting $n_{a}$ bits from Alice to Bob with fidelity $1-\epsilon$. The ancillas are initially in the maximally entangled state $\left|\Phi_{M}\right\rangle_{A^{\prime} B^{\prime}}$ where $\log M$ is the amount of initial entanglement required to assist the communication. Let $|x\rangle_{A}$ carry the $n_{a}$-bit message of Alice. By definition (17) and Uhlmann's theorem [49], the state change is given by

$$
\begin{aligned}
& |x\rangle_{A}|0\rangle_{B}\left|\Phi_{M}\right\rangle_{A^{\prime} B^{\prime}} \stackrel{\mathcal{P}_{t}}{\longrightarrow}\left|\eta_{x}\right\rangle \\
& \left|\eta_{x}\right\rangle=\sqrt{1-\epsilon_{x}}|0\rangle_{A}|x\rangle_{B}\left|c_{x}\right\rangle_{A^{\prime} B^{\prime}}+\sqrt{\epsilon_{x}}\left|e_{x}\right\rangle_{A B A^{\prime} B^{\prime}}
\end{aligned}
$$

where $\operatorname{tr}_{A^{\prime} B^{\prime}}\left|e_{x}\right\rangle\left\langle\left. e_{x}\right|_{A B A^{\prime} B^{\prime}}\right.$ has support orthogonal to $|0\rangle_{A}|x\rangle_{B}$.

In the entanglement generation protocol, Alice inputs half of $\left|\Phi_{2^{n}{ }_{a}}\right\rangle_{A A^{\prime \prime}}$ while Bob still inputs $|0\rangle_{B}$. The input and output states are given by

$$
\begin{aligned}
2^{-n_{a} / 2} \sum_{x}|x\rangle_{A}|x\rangle_{A^{\prime \prime}}|0\rangle_{B}\left|\Phi_{M}\right\rangle_{A^{\prime} B^{\prime}} \\
\left|\eta^{(\epsilon)}\right\rangle=2^{-n_{a} / 2} \sum_{x}\left[\sqrt{1-\epsilon_{x}}|0\rangle_{A}|x\rangle_{B}\left|c_{x}\right\rangle_{A^{\prime} B^{\prime}}\right. \\
\left.+\sqrt{\epsilon_{x}}\left|e_{x}\right\rangle_{A B A^{\prime} B^{\prime}}\right]|x\rangle_{A^{\prime \prime}}
\end{aligned}
$$

$$
\begin{aligned}
& \text { For }\left|\eta^{(0)}\right\rangle= \\
& \begin{aligned}
E\left(\left|\eta^{(0)}\right\rangle\right) & =S\left(\operatorname{tr}_{B B^{\prime}}\left|\eta^{(0)}\right\rangle\left\langle\eta^{(0)}\right|\right) \\
& =S\left(2^{-n_{a}} \sum_{x}|0\rangle\left\langle\left. 0\right|_{A} \mid x\right\rangle\left\langle\left. x\right|_{A^{\prime \prime}} \otimes \operatorname{tr}_{B^{\prime}} \mid c_{x}\right\rangle\left\langle c_{x}\right|\right) \\
& =n_{a}+2^{-n_{a}} \sum_{x} S\left(\operatorname{tr}_{B^{\prime}}\left|c_{x}\right\rangle\left\langle c_{x}\right|\right) \\
& =n_{a}+2^{-n_{a}} \sum_{x} E\left(\left|c_{x}\right\rangle\right) .
\end{aligned}
\end{aligned}
$$

Applying the definition of the entanglement destroying capacity to (36)

$$
\forall_{x} E\left(\left|\eta_{x}\right\rangle\right) \geq \log M-t E_{U^{\dagger}}^{(1, *)}=\log M-t E_{U^{\dagger}}^{(\emptyset)} .
$$

Since

$$
\left\langle0 | _ { A } \left\langle\left. x\right|_{B}\left\langle\left. c_{x}\right|_{A^{\prime} B^{\prime}} \mid \eta_{x}\right\rangle_{A A^{\prime} B B^{\prime}} \geq \sqrt{1-\epsilon}\right.\right.
$$

and

$$
\operatorname{Sch}\left(\left|c_{x}\right\rangle\right) \leq \operatorname{Sch}\left(\left|\eta_{x}\right\rangle\right) \leq M d^{2 t}
$$

Fannes' inequality implies

$$
\forall_{x} \mid E\left(\left|c_{x}\right\rangle\right)-E\left(\left|\eta_{x}\right\rangle\right) \mid \leq 4 \sqrt{\epsilon}[\log M+2 t \log d]+1 / e .
$$

Hence,

$$
\begin{aligned}
& E\left(\left|\eta^{(0)}\right\rangle\right) \geq n_{a}+\log M-t E_{U^{\dagger}}^{(\emptyset)} \\
&-4 \sqrt{\epsilon}[\log M+2 t \log d]-1 / e .
\end{aligned}
$$

Using $\left\langle\eta^{(0)} \mid \eta^{(\epsilon)}\right\rangle \geq \sqrt{1-\epsilon}$, and that

$$
\begin{aligned}
& \operatorname{Sch}\left(\left|\eta^{(\epsilon)}\right\rangle\right) \leq d^{2 t} M \\
& \operatorname{Sch}\left(\left|\eta^{(0)}\right\rangle\right) \leq \sum_{x} \operatorname{Sch}\left(\left|c_{x}\right\rangle\right) \leq 2^{n_{a}} d^{2 t} M
\end{aligned}
$$

Fannes' inequality implies

$$
\begin{aligned}
\mid E\left(\left|\eta^{(0)}\right\rangle\right)-E & \left(\left|\eta^{(\epsilon)}\right\rangle\right) \mid \\
& \leq 2 \sqrt{\epsilon}\left[\log M+2 t \log d+n_{a}\right]+1 / e .
\end{aligned}
$$

Thus,

$$
\begin{aligned}
E\left(\mid \eta^{(\epsilon))}\right) \geq & n_{a}+\log M-t E_{U^{\dagger}}^{(\emptyset)} \\
& -2 \sqrt{\epsilon}\left[3 \log M+6 t \log d+n_{a}\right]-2 / e
\end{aligned}
$$


and

$$
\begin{aligned}
E_{U}^{(\emptyset)} \geq & \frac{1}{t}\left[E\left(\left|\eta^{(\epsilon)}\right\rangle\right)-\log M\right] \\
\geq & \frac{n_{a}}{t}-E_{U^{\dagger}}^{(\emptyset)} \\
& -2 \sqrt{\epsilon}\left[\frac{3}{t} \log M+6 \log d+\frac{n_{a}}{t}\right]+\frac{2}{e t} .
\end{aligned}
$$

In particular, consider the entanglement-assisted communication protocol in Section IV-C. For any $\delta_{\mathcal{E}}>0, \exists \mathcal{E}$ consisting of $M_{0}$-dimensional bipartite states with

$$
\chi(U \mathcal{E})-\chi(\mathcal{E}) \geq \Delta \chi_{U}^{(1, *)}-\delta_{\mathcal{E}}=C_{\rightarrow, U}^{E}-\delta_{\mathcal{E}} .
$$

Following (26), $t=c n+n k$ for some constant $c$ and the rate is $\frac{n_{a}}{t} \geq C_{\rightarrow, U}^{E}-\delta_{\mathcal{E}}-\delta_{n}-\frac{c}{k} C_{\rightarrow, U}^{E}$, where $\delta_{n} \rightarrow 0$ as $n \rightarrow \infty$. The total error is $\epsilon=k \epsilon_{n}$, where $\lim _{n \rightarrow \infty} \epsilon_{n}=0$. The RSP method in [45] can be improved [51] to prepare $n$ states from an ensemble $\mathcal{E}$ with $n \chi\left(\operatorname{tr}_{A A^{\prime}} \mathcal{E}\right)+o(n)$ cbits and $n S\left(\bar{\rho}_{B}\right)+o(n) \leq$ $n \log M_{0}$ ebits where $\bar{\rho}_{B}$ is the average reduced density matrix of the ensemble as seen from Bob, so that $M \leq M_{0}^{t}$. Putting all these parameters into (42)

$$
\begin{aligned}
E_{U}^{(\emptyset)} \geq & C_{\rightarrow, U}^{E}-\left[\delta_{\mathcal{E}}+\delta_{n}+\frac{c}{k} C_{\rightarrow, U}^{E}\right]-E_{U^{\dagger}}^{(\emptyset)} \\
& -2 \sqrt{\epsilon}\left[3 \log M_{0}+6 \log d+\frac{n_{a}}{t}\right]+\frac{2}{e n(c+k)} .
\end{aligned}
$$

For any $\delta>0$, choose

1) $\mathcal{E}$ such that $\delta_{\mathcal{E}}<\delta / 5$,

2) $k$ such that $\frac{c}{k} C_{\rightarrow, U}^{E}<\delta / 5$ and $2 / e(c+k) \leq \delta / 5$ so that $2 / e n(c+k) \leq \delta / 5$

3) $n$ such that $\delta_{n}<\delta / 5$ and $\epsilon=k \epsilon_{n}$ small enough for $2 \sqrt{\epsilon}\left[3 \log M_{0}+6 \log d+\frac{n_{a}}{t}\right]<\delta / 5$.

To summarize, for all $U$, we have

- $C_{\rightarrow, U}, C_{\leftarrow, U} \leq C_{+, U} \leq E_{c, U}^{(1, *)} \leq \operatorname{Sch}(U) \leq 2 \log d$;

- $C_{+, U} \leq C_{\rightarrow, U}+C_{\leftarrow, U}, C_{+, U} \leq E_{U}^{(\emptyset)}, C_{+, U} \leq C_{+, U}^{E}$;

- $C_{\leftarrow, U} \leq C_{\leftarrow, U}^{E} \leq \min \left(2 \log d, E_{U}^{(\emptyset)}+E_{U^{\dagger}}^{(\emptyset)}\right)$

$C_{\rightarrow, U} \leq C_{\rightarrow}^{E}, U \leq \min \left(2 \log d, E_{U}^{(\emptyset)}+E_{U^{\dagger}}^{(\emptyset)}\right)$

$C_{+, U}^{E} \leq C_{\rightarrow, U}^{E}+C_{\leftarrow, U}^{E} \leq \min \left(4 \log d, 2\left(E_{U}^{(\emptyset)}+E_{U^{\dagger}}^{(\emptyset)}\right)\right) ;$

- $C_{\leftrightarrow, U} \leq \min \left(C_{\rightarrow, U}, C_{\leftarrow, U}\right), C_{+, U} \geq 2 C_{\leftrightarrow, U}$.

We now return briefly to Hamiltonian capacities. Recall from Section I-E that any Hamiltonian capacity can be expressed in terms of the corresponding gate capacity

$$
G_{H}=\lim _{s \rightarrow 0} \frac{1}{s} G_{U=e^{-i H s}} .
$$

The finiteness of $G_{H}$ is not immediate from the above definition. Even though $G_{U=e^{-i H s}} \rightarrow 0$ when $s \rightarrow 0$ due to continuity, it is not guaranteed that $G_{U=e^{-i H s}} \leq O(s)$. One may argue that physically, the rate should be finite, but the availability of unlimited local resources complicates the argument. We now provide a proof of the finiteness of the Hamiltonian capacities [52].

Bound 4: Hamiltonian capacities are finite.

Proof: Recall that the entanglement capacity of a gate $U$ is no more than the average amount of entanglement required to simulate $U$ given free classical communication. Reference [6] describes a method to simulate $U=e^{-i \alpha \sigma_{z} \otimes \sigma_{z}}$ with $O(\alpha)$ entanglement, which implies that $E_{H=\sigma_{z} \otimes \sigma_{z}}$ is finite. Reference [39] describes a method to simulate any other Hamiltonian $H^{\prime}$ in $d \times d$ using the Hamiltonian $H=\sigma_{z} \otimes \sigma_{z}$ with $O\left(d^{4}\right)$ overhead, so that $\forall_{H^{\prime}} E_{H^{\prime}} \leq O\left(d^{4}\right) E_{H=\sigma_{z} \otimes \sigma_{z}}$ is also finite.

The finiteness of other capacities now follows. From the summary, $C_{+, H^{\prime}}^{E} \leq 2\left(E_{H^{\prime}}+E_{-H^{\prime}}\right)$ is an upper bound to all other classical capacities of $H^{\prime}$.

\section{BidiReCTIONAL ChANNELS ON $d_{1} \times d_{2}$}

We have assumed $U$ acting on a $d \times d$ bipartite system. We note here that all the results discussed hold for a nonlocal gate (or Hamiltonian) acting on a $d_{1} \times d_{2}$ system (without loss of generality, $d_{1} \leq d_{2}$ ). The interested reader can easily verify that all the arguments hold in this case, because the fact $d_{1}=d_{2}$ is never used in the proofs. We also note a subtle observation, that the $d_{1} \times d_{2}$ case is not described by embedding the operation in a $d_{2} \times d_{2}$ system by taking the direct sum with a $\left(d_{2}-d_{1}\right)$-dimensional identity matrix acting on the side of lower dimension.

\section{OPEN QUESTIONS AND EXAMPLES}

We have found expressions for the entanglement capacity and the entanglement-assisted classical capacity of unitary bidirectional channels, defined classical capacities for them, and provided general bounds for the capacities. We conclude first with a list of open questions, followed by examples to illustrate our results and our open questions.

\section{A. Open Questions}

- How large do the ancillas $A^{\prime} B^{\prime}$ need to be in the optimal input for entanglement generation? How large do $A^{\prime} B^{\prime}$ need to be, and how many states are needed in the optimal ensemble for entanglement-assisted classical communication? These are important for numerical studies of the capacities.

-Will infinite-dimensional ancillas improve the entanglement capacity and the entanglement-assisted one-way classical capacity? Will an ensemble with an infinite number of members improve the latter?

We have learned of two interesting results on entanglement capacity involving infinite-dimensional systems or ancillas since the initial submission of this paper. First, Eisert [53] has proved that under certain restrictions on the system energy, infinite dimensional ancillas are no better than finite-dimensional ones for generating entanglement. Second, van Enk [54] has found a Hamiltonian acting on infinite-dimensional systems that creates an infinite amount of entanglement in an infinitely short time (compare to our proof for finiteness of all Hamiltonian capacities in the finite-dimensional case).

- How do the forward and backward rates trade off with each other (in either the unassisted or assisted case)?

- Are forward and backward classical capacities always equal (in either the unassisted or assisted case)?

- Is there a gate $U$ with $C_{+, U}<E_{e, U}^{(\emptyset)}$ a strict inequality?

- Is $E_{e, U}^{(1, *)}=E_{e, U^{\dagger}}^{(1, *)}$ for all $U$ ? Both quantities relate to how entangling a nonlocal gate is. However, we can only prove the equality when $U=U^{T}$, by using the fact $E_{e, U}^{(1, *)}=E_{e, U^{*}}^{(1, *)}$ 
( $U^{*}$ is the complex conjugate of $U$ ). This generalizes the proof in [50] for 2-qubit gates since $U=U^{T}$ for all 2-qubit gates in their normal form [8]. Numerical work suggests that the equality does not hold for some $U$ in higher dimensions [55].

- When can a gate be simulated efficiently, i.e., by an amount of some resource equal to the capacity?

- How do auxiliary resources of quantities linear in the number of uses affect the capacity?

\section{B. Examples}

Example 1: Let $U=$ CNOT. It can be simulated using 1 ebit and 1 bit of classical communication in each direction [9]. Thus, $E_{\mathrm{CNOT}}^{(\emptyset)} \leq 1, C_{\rightarrow}^{E}, \mathrm{CNOT} \leq 1, C_{\leftarrow, \mathrm{CNOT}}^{E} \leq 1$, and Bound 2 further implies $C_{+}$, CNOT $\leq 1$. These are all achievable with obvious methods, without the need of entanglement assistance in $C_{\rightarrow}^{E}$, CNOT and $C_{\leftarrow}^{E}, \mathrm{CNOT}$ and without the need of initial entanglement in $E_{\mathrm{CNOT}}^{(\emptyset)}$. Therefore,

$$
\begin{aligned}
C_{\rightarrow, \mathrm{CNOT}}=C_{\leftarrow, \mathrm{CNOT}}=C_{+, \mathrm{CNOT}}=C_{\rightarrow}^{E}, \mathrm{CNOT} \\
\quad=C_{\leftarrow, \mathrm{CNOT}}^{E}=E_{\mathrm{CNOT}}^{(\emptyset)}=E_{\mathrm{CNOT}}^{(1, \emptyset)}=1 .
\end{aligned}
$$

The rate pairs in the triangle with vertices $(0,0),(0,1),(1,0)$ are achievable without entanglement assistance, and convexity implies no other pair is achievable. We also have $C_{+, \mathrm{CNOT}}^{E} \geq 2$ due to the following protocol. Starting with the EPR state $\left|\Phi_{2}\right\rangle_{A B}$, Alice applies $\sigma_{x}^{a}$ and Bob applies $\sigma_{z}^{b}$ if their respective input bits are $a$ and $b$. The CNOT is then applied, converting the state to $\frac{(-1)^{a b}}{\sqrt{2}}\left(|0\rangle+(-1)^{b}|1\rangle\right)|a\rangle$. Thus, rate pairs in the square with vertices $(0,0),(0,1),(1,0),(1,1)$ are all achievable with entanglement assistance, and by monotonicity, no other rate pair is achievable.

Example 2: For $U=$ SWAP, we have the general upper bounds $E_{\text {SWAP }}^{(\emptyset)} \leq 2, C_{\rightarrow \text {, SWAP }}^{E} \leq 2, C_{\leftarrow \text {, SWAP }}^{E} \leq 2$, and Bound 2 implies $C_{+}$, SWAP $\leq 2$. These are all achievable as follows. $E_{\mathrm{SWAP}}^{(\emptyset)}=E_{\mathrm{SWAP}}^{(1, \not)}$ is achieved on the input $\left|\Phi_{2}\right\rangle_{A A^{\prime}}\left|\Phi_{2}\right\rangle_{B B^{\prime}}$. To achieve the forward assisted classical capacity, Alice and Bob start with the state $\left|\Phi_{2}\right\rangle_{A B^{\prime \prime}}\left|\Phi_{2}\right\rangle_{B B^{\prime}}$ and Alice applies $\sigma_{x A}^{a_{1}} \sigma_{z A}^{a_{2}}$ when her 2-bit message is $a_{1} a_{2}$. Then SWAP is applied. In other words, superdense coding [17] is performed, consuming an existing EPR pair on $A B^{\prime \prime}$, while a new EPR pair is created on $A B^{\prime}$ simultaneously. Thus, the unassisted and assisted one-way classical capacities are both 2. $C_{+}$, SWAP $=2$ is achieved in the obvious way. Superdense coding in both the forward and backward directions implies $C_{+, \text {SWAP }}^{E} \geq 4$, and by the monotonicity of the achievable region of assisted rate pairs, $C_{+}^{E}$, SWAP $=4$. Therefore, any rate pair inside the triangle with vertices $(0,0),(0,2),,(2,0)$ can be achieved without entanglement assistance, and any rate pair inside the square with vertices $(0,0),(0,2),(2,0),(2,2)$ can be achieved with entanglement assistance.

The CNOT and SWAP are very simple. We now turn to more intriguing examples.

Example 3: The gate $\mathrm{J}$ acts as

$$
|00\rangle \leftrightarrow \frac{1}{\sqrt{2}}(|00\rangle+|11\rangle), \quad|01\rangle \leftrightarrow|01\rangle
$$

$$
|11\rangle \leftrightarrow \frac{1}{\sqrt{2}}(|00\rangle-|11\rangle), \quad|10\rangle \leftrightarrow|10\rangle
$$

where the first and second registers are $A$ and $B$ (same throughout the examples). Without ancillas, J creates 1 ebit but seems to create less than 1 cbit in 1-shot, but [25] presents a product 2-qubit input that communicates $1 \mathrm{cbit}$ from Alice to Bob.

Numerical optimization of the generated entanglement with two-dimensional $A^{\prime}$ and $B^{\prime}$ in (7) is 1.83186 ebits, and the optimal input has 0.055338 ebit. As a comparison, only 1.8113 ebits is generated by inputting $\left|\Phi_{2}\right\rangle_{A A^{\prime}}\left|\Phi_{2}\right\rangle_{B B^{\prime}}$.

Starting from $\left|\Phi_{2}\right\rangle_{A B}=\frac{1}{\sqrt{2}}(|00\rangle+|11\rangle)$, Alice and Bob can communicate 1 bit to each other, by applying $\sigma_{z}^{a}$ and $\sigma_{z}^{b}$ if their respective messages are $a$ and $b$. The J gate further converts the state to $|x\rangle_{A}|x\rangle_{B}$ where $x=a+b \bmod 2$, from which they learn each other's input.

We suspect $C_{+}, J<E_{\mathrm{J}}^{(\emptyset)}$. For instance, the best total rate we found requires creating 1 ebit with one use of $\mathrm{J}$ followed by assisted two-way communication in the second use of J. Asymptotically, 2.83186 uses of J can create at least $1.83186 \times 2$ bits of communication, so that $C_{+}, \mathrm{J} \geq 1.2938$, which is much less than 1.83186.

Example 4: Denote the "cyclic permutation" gate by CP. It acts as

$$
\begin{array}{llrl}
|x\rangle|y\rangle \rightarrow|x\rangle|y-1\rangle, & & \text { if } y \neq 0 \\
|x\rangle|y\rangle \rightarrow|x-1\rangle|y-1\rangle, & & \text { if } y=0
\end{array}
$$

where \pm is modulo $d$. $\mathrm{Sch}(\mathrm{CP})=2$ for all $d$, thus, $E_{\mathrm{CP}}^{(\emptyset)} \leq 1$. This is achievable on the input $\frac{1}{\sqrt{2}}|0\rangle_{A}(|0\rangle+|1\rangle)_{B} . C_{\leftarrow, \mathrm{CP}} \geq 1$ since Bob can send 1 bit $b \in\{0,1\}$ to Alice with the input $|0\rangle_{A}|b\rangle_{B}$. Thus,

$$
\forall_{d} E_{\mathrm{CP}}^{(\emptyset)}=E_{\mathrm{CP}}^{(1, \emptyset)}=C_{\leftarrow, \mathrm{CP}}=C_{+, \mathrm{CP}}=1 .
$$

For $d=3$, we have also studied forward communication without ancillas. It is impossible to transmit 1 bit from Alice to Bob by one use of $U$, but it is possible asymptotically, so that $C \rightarrow, \mathrm{CP}=1$.

Example 5: Define the gate $\mathrm{AE}$ on $d \times d$ by

$$
\begin{array}{lll}
\forall x & |x 0\rangle \leftrightarrow|x x\rangle \\
\forall 0 \neq y \text { and } y \neq x & |x y\rangle \leftrightarrow|x y\rangle .
\end{array}
$$

Since $\operatorname{Sch}(U)=d, E_{\mathrm{AE}}^{(\emptyset)} \leq \log d$, and this is achievable on the input $\sum_{x}|x\rangle_{A}|0\rangle_{B}$, and $E_{\mathrm{AE}}^{(\emptyset)}=E_{\mathrm{AE}}^{(1, \emptyset)}=\log d . C_{\rightarrow, \mathrm{AE}}=$ $\log d$ is achievable in the obvious manner. Thus, $C_{+}, \mathrm{AE}=$ $\log d$. We can prove that one use of AE can communicate strictly less than $\log d$ cbit from Bob to Alice starting from product states but allowing ancillas. However, we suspect $C_{\leftarrow, \mathrm{AE}}<$ $C_{\rightarrow, \mathrm{AE}}=\log d$.

Example 6: Since the initial submission of this paper, Childs, Leung, Verstraete, and Vidal [56] have analytically proved that the asymptotic entanglement capacity of any Hamiltonian locally equivalent to $\alpha \sigma_{x} \otimes \sigma_{x}+\beta \sigma_{y} \otimes \sigma_{y}$ can be achieved without ancillas, and the capacity is $\approx 1.9123(\alpha+\beta)$ following [7]. 


\section{APPENDIX}

\section{LINEAR BOUND IN COMMUNICATION COST FOR DISTILLATION}

In this appendix, we obtain a bound on the communication cost in distillation using [21] which derives the enhancement factor of the capacity of a noiseless quantum channel assisted by noisy entanglement, i.e., unlimited supply of the mixed state $\rho$.

Suppose given $\rho^{\otimes q n}$, $c n$ forward classical bits (in either direction) is sufficient to distill $n$ ebits $(q \geq 1 / D(\rho))$. Here, we do not require maximum yield of entanglement, so that the classical communication cost is upper-bounded by that required in the more difficult job of distillation.

Then, the following is a noisy superdense coding strategy for Alice and Bob-first distill and then perform noiseless superdense coding

$$
\begin{gathered}
c n \text { cbits }+\rho^{\otimes q n} \rightarrow n \text { ebits } \\
n \text { ebits }+n \text { qubits } \rightarrow 2 n \text { cbits. }
\end{gathered}
$$

Together, the enhancement factor is equal to $\frac{2 n-c n}{n}=2-c$, which cannot exceed the optimal value [21]

$$
C_{s d}=1+\sup _{n} \sum_{\Lambda_{A}} \frac{n S\left(\operatorname{tr}_{A}(\rho)\right)-S\left(\Lambda_{A}\left(\rho^{\otimes n}\right)\right)}{S\left(\Lambda_{A}\left(\operatorname{tr}_{B}\left(\rho^{\otimes n}\right)\right)\right.}
$$

where the supremum is taken over all TCP maps $\Lambda_{A}$ on Alice's half of $\rho^{\otimes n}$. Hence, $c \geq 2-C_{s d} \equiv \Delta$. Even though it is not known how to calculate $\Delta$ for an arbitrary $\rho$ it is unlikely to be zero for all $\rho$. If $\exists \rho$ for which $\Delta \neq 0$, then distillation would take at least linear classical communication.

\section{ACKNOWLEDGMENT}

We wish to thank M. Leifer, L. Henderson, and N. Linden for discussions and for kindly sharing their results on entanglement capacity prior to publication. We also thank the above, as well as L. Spector and H. Bernstein, and K. Hammerer, G. Vidal, and J. I. Cirac for communicating their results on classical communications with bidirectional channels.

We are indebted to many colleagues for their inputs to our work. We thank P. Shor for communicating his RSP results which are crucial to our results. We thank A. Childs and H.-K. Lo for their critical reading of the manuscript and for many constructive suggestions, part of which motivated a more precise version of Theorem 1 and the problem on $d_{1} \times d_{2}$ systems. The finiteness of the Hamiltonian capacities was questioned by G. Vidal, who also provided the proof for the finiteness of entanglement capacity. We wish to thank M. Nielsen for his upper bound on the entanglement capacity in terms of the Schmidt number. We wish to thank I. Devetak for important input in proving Bound 2; D. DiVincenzo, J. Dodd, J. Eisert, A. Kitaev, B. Terhal, and other members of the IQI at Caltech for additional helpful discussions.

Since this paper was first posted, other related results have been posted [50], [56], [57], [58].

\section{REFERENCES}

[1] C. H. Bennett, S. Braunstein, I. L. Chuang, D. P. DiVincenzo, D. Gottesman, J. A. Smolin, B. M. Terhal, and W. K. Wootters, "Unpublished discussion during the 6th Quantum Computation Workshop," ISI, Torino, Italy, 1998.

[2] C. H. Bennett and D. Gottesman, private communication, 1998.

[3] J. Eisert, K. Jacobs, P. Papadopoulos, and M. B. Plenio, "Optimal local implementation of non-local quantum gates," Phys. Rev. A, vol. 62, 2000. 052317, arXive e-print quant-ph/0005101v1.

[4] D. Collins, N. Linden, and S. Popescu, arXive e-print quant$\mathrm{ph} / 0005102 \mathrm{v} 1$.

[5] P. Zanardi, C. Zalka, and L. Faoro, arXive e-print quant-ph/0005031.

[6] J. I. Cirac, W. Dur, B. Kraus, and M. Lewenstein, "Entangling operations and their implementation using a small amount of entanglement," Phys. Rev. Lett., vol. 86, p. 544, 2001. arXive e-print quant-ph/0007057.

[7] W. Dur, G. Vidal, J. I. Cirac, N. Linden, and S. Popescu, "Entanglement capabilities of non-local Hamiltonians," Phys. Rev. Lett., vol. 87, 2001. 137901. arXive e-print quant-ph/0006034.

[8] B. Kraus and J. I. Cirac, arXive e-print quant-ph/0011050.

[9] D. Gottesman, Group 22: Proc. XXII Int. Colloquium on Group Theoretical Methods in Physics, S. P. Corney, R. Delbourgo, and P. D. Jarvis, Eds. Cambridge, MA: International, 1999, pp. 32-33. arXive e-print quant-ph/9807006.

[10] A. S. Holevo, "The capacity of the quantum channel with general signal states," IEEE Trans. Inform. Theory, vol. 44, pp. 269-273, Jan. 1998.

[11] B. Schumacher and M. D. Westmoreland, "Sending classical information via noisy quantum channels," Phys. Rev. A, vol. 56, pp. 131-138, 1997.

[12] C. H. Bennett, P. W. Shor, J. A. Smolin, and A. V. Thapliyal, arXive e-print quant-ph/0106052.

[13] A. S. Holevo, arXive e-print quant-ph/0106075.

[14] M. A. Nielsen and I. L. Chuang, Quantum Computation and Quantum Information. Cambridge, U.K.: Cambridge Univ. Press, 2000.

[15] C. H. Bennett, D. P. DiVincenzo, J. A. Smolin, and W. K. Wootters, "Mixed state entanglement and quantum error correction," Phys. Rev. A, vol. 54, p. 3824, 1996. arXive e-print quant-ph/9604024.

[16] M. Leifer, L. Henderson, and N. Linden, arXive e-print quant$\mathrm{ph} / 0205055$.

[17] C. H. Bennett and S. J. Wiesner, "Communication via one- and twoparticle operators on Einstein-Podolsky-Rosen states," Phys. Rev. Lett., vol. 69, pp. 2881-2884, 1992.

[18] S. Bose, M. B. Plenio, and B. Vedral, "Mixed state dense coding and its relation to entanglement measures," J. Mod. Opt., vol. 47, p. 291, 2000. arXive e-print quant-ph/9810025.

[19] T. Hiroshima, arXive e-print quant-ph/0009048.

[20] G. Bowen, "Classical information capacity of superdense coding," Phys. Rev. A, vol. 63, 2001. 022302, arXive e-print quant-ph/0101117.

[21] M. Horodecki, P. Horodecki, R. Horodecki, D. Leung, and B. Terhal, "Classical capacity of a noiseless quantum channel assisted by noisy entanglement," Quantum Inform. Comput., vol. 1, p. 78, 2001.

[22] C. E. Shannon, "Two-way communication channels," in Proc. 4th Berkeley Symp. Math. Stat. Prob. Berkeley, CA: Univ. Caligornia Press, 1961.

[23] K. Hammerer, G. Vidal, and J. I. Cirac, arVix quant-ph/0205100.

[24] M. Leifer, L. Henderson, and N. Linden, private communication, 2002.

[25] L. Spector and H. Bernstein, "Poster presentation," presented at the 6th Int. Conf. Quantum Computation, Measurement, and Communications, Cambridge, MA, July 2002, personal communication.

[26] M. J. Donald, M. Horodecki, and O. Rudolph, arXive e-print quant$\mathrm{ph} / 0105017$.

[27] G. Vidal, "Entanglement monotones," J. Mod. Opt., vol. 47, p. 355, 2000. arXive e-print quant-ph/9807077.

[28] P. M. Hayden, M. Horodecki, and B. M. Terhal, "The asymptotic entanglement cost of preparing a quantum state," J. Phys. A: Math. Gen., vol. 34, no. 35, pp. 6891-6898, 2001. arXive e-print quant-ph/0008134.

[29] D. Jonathan and M. B. Plenio, "Entanglement-assisted local manipulation of pure quantum states," Phys. Rev. Lett., vol. 83, p. 1455, 1999. arXive e-print quant-ph/9903054.

[30] P. W. Shor, J. A. Smolin, and B. M. Terhal, "Nonadditivity of bipartite distillable entanglement follows from conjecture on bound entangled Werner states," Phys. Rev. Lett., vol. 86, pp. 2681-2684, 2001, arXive e-print quant-ph/0010054

[31] C. H. Bennett, H. J. Bernstein, S. Popescu, and B. Schumacher, "Concentrating partial entanglement by local operations," Phys. Rev. A, vol. 53, pp. 2046-2052, 1996. arXive e-print quant-ph/9511030. 
[32] H.-K. Lo and S. Popescu, "The classical communication cost of entanglement manipulation: Is entanglement an inter-convertible resource?," Phys. Rev. Lett., vol. 83, pp. 1459-1462, 1999. arXive e-print quant$\mathrm{ph} / 9902045$.

[33] H.-K. Lo, "Classical-communication cost in distributed quantum-information processing: A generalization of quantum-communication complexity," Phys. Rev. A, vol. 62, 2000. 012313. arXive e-print quant-ph/9912009.

[34] P. Hayden and A. Winter, arXive e-print quant-ph/0204092.

[35] A. Harrow and H.-K. Lo, arXive e-print quant-ph/0204096.

[36] M. A. Nielsen, "Quantum information theory," $\mathrm{PhD}$ dissertation, Univ. New Mexico, Albuquerque, NM, 1998.

[37] B. M. Terhal, M. Horodecki, D. W. Leung, and D. P. DiVincenzo, "The entanglement of purification," J. Math. Phys., vol. 43, p. 4286, 2002. arXive e-print quant-ph/0202044.

[38] B. M. Terhal, private communication, 2001

[39] C. H. Bennett, J. I. Cirac, M. S. Leifer, D. W. Leung, N. Linden, S Popescu, and G. Vidal, "Optimal simulation of two-qubit Hamiltonians using general local operations," Phys. Rev. A, vol. 66, 2002. 012305. arXive e-print quant-ph/0107035.

[40] C. H. Bennett, P. W. Shor, and A. Winter, in preparation.

[41] P. Hausladen, R. Jozsa, B. Schumacher, M. Westmoreland, and W. Wootters, "Classical information capacity of a quantum channel," Phys. Rev. A, vol. 54, p. 1869, 1996.

[42] A. S. Holevo, "Bounds for the quantity of information transmitted by a quantum communication channel," Probl. Inform. Transm., vol. 9, pp. 177-183, 1973.

[43] C. H. Bennett, D. P. DiVincenzo, P. W. Shor, J. A. Smolin, B. M. Terhal, and W. K. Wootters, "Remote state preparation," Phys. Rev. Lett., vol. 87, 2000. 077902. arXive e-print quant-ph/0006044.
[44] I. Devetak and T. Berger, arXive e-print quant-ph/0102123.

[45] P. W. Shor, private communication, 2001.

[46] C. H. Bennett, P. Hayden, D. W. Leung, and A. Winter, in preparation

[47] M. Fannes, Commun. Math. Phys., vol. 31, p. 291, 1973.

[48] M. A. Nielsen, arXive e-print quant-ph/9908086.

[49] A. Uhlmann, "The 'transition probability' in the state space of a *-algebra," Rep. Math. Phys., vol. 9, pp. 273-279, 1976.

[50] D. W. Berry and B. C. Sanders, arXive e-print quant-ph/0205181.

[51] C. H. Bennett, P. M. Hayden, D. W. Leung, P. W. Shor, and A. Winter, in preparation.

[52] The question of finiteness of Hamiltonian capacity and a proof for the entanglement capacity are due to G. Vidal, personal communication, 2002.

[53] J. Eisert, "The restriction to enable the result to hold is that, $\operatorname{tr} e^{-\beta H}$ and $\operatorname{tr}\left(\operatorname{tr}_{B B^{\prime}}|\psi\rangle\left\langle\left.\psi\right|_{A B A^{\prime} B^{\prime}} H\right)\right.$ are both finite for some energy operator $H$," personal communication, 2003.

[54] S. J. van Enk, arXive e-print quant-ph/0302086.

[55] A. M. Childs, D. W. Leung, and J. A. Smolin, private communication, 2002.

[56] A. M. Childs, D. W. Leung, F. Verstraete, and G. Vidal, arXive e-print quant-ph/0207052.

[57] D. W. Berry and B. C. Sanders, arXive e-print quant-ph/0207065.

[58] C. M. Dawson, J. L. Dodd, A. Gilchrist, D. Mortimer, T. J. Osborne, M. J. Bremner, A. W. Harrow, A. Hines, and M. A. Nielsen, arXive e-print quant-ph/0208077. 